\title{
Philosophiques
}

\section{Bosanquet, Collingwood et l'esthétique idéaliste britannique}

\section{Chinatsu Kobayashi}

Volume 36, numéro 1, printemps 2009

L'idéalisme britannique

URI : https://id.erudit.org/iderudit/038014ar

DOI : https://doi.org/10.7202/038014ar

Aller au sommaire du numéro

Éditeur(s)

Société de philosophie du Québec

ISSN

0316-2923 (imprimé)

1492-1391 (numérique)

Découvrir la revue

Citer cet article

Kobayashi, C. (2009). Bosanquet, Collingwood et l'esthétique idéaliste

britannique. Philosophiques, 36(1), 149-182. https://doi.org/10.7202/038014ar

\section{Résumé de l'article}

Après un bref survol de l'esthétique britannique au $\mathrm{xx}^{\mathrm{e}}$ siècle, les objections de Wollheim à la théorie « idéelle » de l'art, qu'il attribue à Croce et à Collingwood, sont présentées. Dans une deuxième partie, les critiques de Bosanquet à

l'endroit de la théorie de Croce sont examinées, pour en conclure qu'on ne peut pas lui attribuer la théorie "idéelle ». Il en va de même pour Collingwood, dont les grandes lignes de son esthétique sont présentées dans la troisième partie, dont le but accessoire est de montrer que sa théorie est par ailleurs plus satisfaisante que celle de Bosanquet ; elle est en réalité très proche de celle de Wollheim, malgré les dires de ce dernier. Dans la conclusion, on revient sur le motif des théories de l'art des idéalistes britanniques, à savoir le rôle social que doit jouer l'oeuvre d'art, pour montrer toute l'actualité de la théorie de Collingwood, pour qui l'oeuvre d'art est le produit non pas de l'artiste seul, mais de l'artiste et de son audience.
Ce document est protégé par la loi sur le droit d'auteur. L'utilisation des services d’Érudit (y compris la reproduction) est assujettie à sa politique d'utilisation que vous pouvez consulter en ligne.

https://apropos.erudit.org/fr/usagers/politique-dutilisation/ 


\title{
Bosanquet, Collingwood et l'esthétique idéaliste britannique
}

\author{
CHINATSU KOBAYASHI \\ Université du Québec à Montréal
}

\begin{abstract}
RÉSUMÉ. - Après un bref survol de l'esthétique britannique au $\mathrm{xx}^{\mathrm{e}}$ siècle, les objections de Wollheim à la théorie «idéelle» de l'art, qu'il attribue à Croce et à Collingwood, sont présentées. Dans une deuxième partie, les critiques de Bosanquet à l'endroit de la théorie de Croce sont examinées, pour en conclure qu'on ne peut pas lui attribuer la théorie «idéelle». Il en va de même pour Collingwood, dont les grandes lignes de son esthétique sont présentées dans la troisième partie, dont le but accessoire est de montrer que sa théorie est par ailleurs plus satisfaisante que celle de Bosanquet; elle est en réalité très proche de celle de Wollheim, malgré les dires de ce dernier. Dans la conclusion, on revient sur le motif des théories de l'art des idéalistes britanniques, à savoir le rôle social que doit jouer l'œuvre d'art, pour montrer toute l'actualité de la théorie de Collingwood, pour qui l'œuvre d'art est le produit non pas de l'artiste seul, mais de l'artiste et de son audience.
\end{abstract}

ABSTRACT. - After a brief survey of British aesthetics in the $X X^{\text {th }}$ century, Wollheim's objections to the 'ideal' theory, which he imputes to Croce and Collingwood, are presented. In the second part, Bosanquet's critique of Croce's theory is presented in order to show that one cannot attribute to him the 'ideal' theory. The same goes for Collingwood, whose aesthetic theory is sketched in the third part, which argues accessorily that it is more satisfactory than Bosanquet's; it is in fact, his own claims to the contrary notwithstanding, rather close to Wollheim's. The conclusion comes back to the central motivation for British Idealist theories of art, namely that the work of art has a social role to play, in order to show the actuality of Collingwood, who viewed the work of art not as the product of the artist alone, but from the artist and the audience alike.

\section{Introduction: l'esthétique en Grande-Bretagne au $\mathbf{x x}^{\mathrm{e}}$ siècle}

Bien que la Grande-Bretagne, de Lord Shaftesbury à Edmund Burke, soit le pays de quelques-unes des premières contributions à l'esthétique comprise comme discipline philosophique indépendante et qu'elle soit le pays de John Ruskin, qui était probablement le critique d'art le plus grand et le plus influent du XIX $^{e}$ siècle $^{1}$, ce domaine de recherche n'a suscité que peu d'intérêt dans

1. Rappelons que Ruskin fit sa réputation grâce à sa prise de position en faveur de Turner et que, par ses écrits, il joua un rôle décisif dans l'émergence et la popularité de "pré-Raphaélites » en peinture, du mouvement néogothique en architecture ainsi que, par le biais de son influence sur le «Arts and Crafts» de William Morris, sur l'ensemble de l'Art nouveau. Mais tous ces mouvements furent rejetés par le «modernisme» du début du $\mathrm{xx}^{\mathrm{e}}$ siècle, et la réputation de Ruskin s'étiola rapidement. 
les cercles philosophiques du tournant $\mathrm{du} \mathrm{xx}^{\mathrm{e}}$ siècle ${ }^{2}$. On doit évidemment distinguer ici entre l'esthétique comprise comme discipline philosophique et la critique d'art, domaine dans lequel les Anglais ont apporté plusieurs contributions importantes au début du $\mathrm{Xx}^{\mathrm{e}}$ siècle; on se rappellera ici les membres du groupe Bloomsbury tels que Roger Fry ou Clive Bell ${ }^{3}$. Comme l'a déjà indiqué John Passmore (Passmore, 1976, 35), pendant les vingt-huit années durant lesquelles G. F. Stout fut son éditeur, la revue Mind n'a publié qu'une poignée d'articles en esthétique. La philosophie britannique était alors divisée entre «idéalistes» et "réalistes». Seulement quatre représentants de l'école «idéaliste », qui était alors dominante, ont manifesté un quelconque intérêt pour l'esthétique ${ }^{4}$. Parmi ceux-ci, John Alexander Smith, qui détenait la Waynflete Chair of Metaphysics à Oxford (ce qui fait de lui un prédécesseur de Collingwood, Ryle et Strawson), ne publia pas ses travaux - ils demeurent inédits aujourd'hui encore - et un autre, son étudiant Arthur Richie Lord, émigra en Afrique du Sud et semble avoir joué uniquement un rôle marginal. Les deux autres, Bernard Bosanquet et Robin Collingwood, semblent donc être les seuls esthéticiens idéalistes d'importance dans la première moitié du vingtième siècle ${ }^{5}$. C'est Collingwood qui, des deux, s'y connaissait le plus en art, et il était du reste beaucoup plus original, ayant produit une philosophie de l'art qui abordait réellement les préoccupations artistiques de son époque dans The Principles of Art (Collingwood, 1938), tandis que l'œuvre principale de Bosanquet, son History of Aesthetics (Bosanquet, 1904), n'était

2. Le lecteur aura compris que je ne discuterai pas des contributions américaines à l'esthétique de langue anglaise, c'est-à-dire des travaux de George Santayana, John Dewey, Suzanne Langer, etc.

3. Voir par exemple (Bell, 1914), dont le concept de "significant form» constitue une contribution fondamentale au «formalisme». Mentionnons également que certains psychologues, à l'instar de James Sully, avaient un intérêt plus que passager pour l'esthétique.

4. Voir Sweet, 2001, pour un tour d'horizon.

5. Les principaux travaux de Bosanquet en esthétique sont Bosanquet, 1904 et 1915. Je vais ici me concentrer sur sa critique de Croce dans Bosanquet, 1920a et 1920b (ce dernier article est une réponse à Carr, 1920, où ce dernier tente de réfuter certaines objections de Bosanquet à l'endroit de Croce). Son History of Æsthetics a un spectre plutôt étroit, mais ce livre est précieux en ce qu'il représente l'unique discussion anglaise (à l'époque) de la tradition esthétique issue de l'idéalisme allemand. Comme Bosanquet le remarqua plus tard à propos de son livre: "C'était simplement la conception hégélienne des faits. Je la considérais, et la considère encore aujourd'hui, évidente et vraie. Je m'en suis tenu simplement à mettre des points sur les $i$ et à barrer les $t$ d'une simple intuition» (Bosanquet, 1920b, 212). Voir Dewey, 1893, et Sully, 1893, pour des comptes rendus critiques qui identifient cette inclination comme sa faiblesse principale. En ce qui concerne Collingwood, je vais me pencher sur The Principles of Art (Collingwood, 1938), négligeant ainsi les Outlines of a Philosophy of Art, de registre plus large, plus hégélien, et moins riche en contenu original (Collingwood, 1925). Ses principaux articles sur l'esthétique ont été réunis dans Collingwood, 1964. Voir aussi les écrits mineurs recueillis dans Collingwood 1995, 1996. 
guère qu'un commentaire de la tradition idéaliste allemande (Hegel, Winckelman, etc.) dont le propos était déjà fort éloigné des exemples concrets ${ }^{6}$. Du côté des "réalistes ", ce domaine de recherche était aussi peu populaire: il n'y eut que Samuel Alexander et E. F. Carritt qui montrèrent un intérêt pour l'esthétique ${ }^{7}$. Alexander élabora dans Beauty and other Forms of Value (Alexander, 1933) et quelques articles réimprimés dans Philosophical and Literary Pieces (Alexander, 1939), une théorie "réaliste» selon laquelle «l'expérience artistique n'est pas de l'ordre de l'imagination mais de la découverte" (Alexander, 1939, 228). De son côté, Carritt enseigna à Oxford jusqu'à la fin des années 1940, mais il abandonna son « réalisme » sous l'influence de l'esthétique du néo-hégélien italien Benedetto Croce. Cependant, il n'atteignit jamais une position originale propre à lui ${ }^{8}$. À titre d'illustration, on peut citer ce passage d'un de ses derniers articles sur Croce:

Je suis sûr qu'à l'époque ou mes pensées n'etaient pas encore reconstruites et réfléchies [unregenerate days], lorsque je disais d'une fleur ou d'une image qu'elle est belle, je voulais dire par là qu'elle possédait cette qualité tout autant que sa forme. Mais j'en suis venu à penser, avec l'aide de Croce, que j'avais tort et que tout ce que je puis dire, c'est qu'elle stimule en moi, et peut-être chez d'autres, une sorte d'expérience que j'appelle esthétique; faire ou imaginer une belle chose, ou en apprécier une, c'est exprimer cette expérience (Carritt 1953, 455).

Dans cet article, je vais me concentrer sur Bosanquet et Collingwood. De nos jours, presque personne n'a entendu parler d'eux, et ce particulièrement à l'extérieur du monde anglophone. Par exemple, si on ouvre l'Encyclopaedia of Aesthetics, publiée en 1998, on trouvera une entrée sur Collingwood (Anderson, 1998), mais on ne trouvera aucune trace de Bosanquet. Les rares personnes qui connaissent ces auteurs les disqualifient habituellement en les qualifiant d'«idéalistes » ou de "néo-hégéliens ». Comme nous le verrons,

6. Collingwood était issu d'une famille remarquablement artistique: son père, romancier, historien et archéologue, était le secrétaire de Ruskin, dont il écrivit une biographie. Collingwood jouait du piano et il mit aussi à profit son talent de dessinateur dans ses travaux d'archéologie. Voir Inglis, 2009, pour une biographie.

7. Il y a certes d'autres figures mineures, comme Louis Arnauld Reid, professeur à Liverpool et plutôt "non aligné », qui publia A Study in Æsthetics en 1931 (Reid, 1931). Pour le compte rendu de cet ouvrage par Collingwood, voir Collingwood, 1995, 208-212.

8. Cela peut être surprenant, mais les néo-hégéliens italiens ont exercé une forte influence à Oxford dans les années 1920 et 1930, y compris sur des figures telles que E. F. Carritt et H. J. Paton, et, dans une certaine mesure seulement, sur Collingwood. Voir Carritt, 1932, 1939. Carritt publia également une anthologie en 1931, The Philosophy of Beauty (Carritt, 1931). Voir Saw, 1963, sur Carritt. En ce qui concerne Croce, voir son Estetica come scienza dell'espressione e linguistica generale (Croce, 1958) que je discuterai plus loin. Ce livre fut traduit en anglais une première fois dans Croce, 1922, et la première partie théorique de ce livre a été retraduite et publiée dans Croce, 1992 (dont je me suis servi), et par la suite en français dans Croce, 2006. (Cette traduction française est partielle et son plan ne correspond pas à celui de Croce, sans qu'aucune indication de cela ne soit fournie; pour autant que je puisse en juger, aucun des passages que je cite se retrouve dans cette traduction. Pour chaque citation, j’ai donné la référence de l'original italien et de la traduction anglaise, et la traduction française a été faite à partir de l'original italien.) 
Collingwood est tout simplement mis à l'écart en tant que représentant d'une théorie «idéelle» de l'art considérée indéfendable, et son rôle réel ou potentiel dans les débats d'aujourd'hui est carrément ignoré. L'historien de l'art Michael Baxandall, souvent célébré pour sa conception de l'œuvre d'art comme solution à un problème émergeant d'une situation particulière causée par l'implémentation d'un ensemble de conventions dans un contexte donné, en est un exemple frappant. Yves Michaud va même jusqu'à décrire les travaux de Baxandall comme "les illustrations les plus concrètes" de la position de Wittgenstein (Michaud, 1999, 85). En fait, Baxandall s'est inspiré directement de Collingwood et non de Wittgenstein'. Michaud attribue aussi à Richard Wollheim l'idée selon laquelle l'art devrait être défini non pas simplement en partant du point de vue de l'artiste, ou du point de vue du spectateur, mais en partant du fait que l'artiste et le spectateur peuvent échanger leur position (Michaud, 1999, 12). Encore une fois, c'est en fait la position de Collingwood ${ }^{10}$, comme nous le verrons, et il y a une certaine ironie dans le fait que cette position soit attribuée à celui qui a tout fait pour s'assurer que les livres de Collingwood ne soient plus jamais lus, comme nous allons aussi le voir.

Après la Seconde Guerre mondiale, l'esthétique a presque disparu des universités britanniques sous l'influence de la philosophie analytique. Si la philosophie se nourrit de tentatives de résoudre des problèmes, voire des paradoxes, la "philosophie du langage ordinaire» et son ennemi le "positivisme logique » avaient au moins ceci en commun qu'ils cherchèrent plutôt à faire disparaître ces problèmes par l'analyse du langage plutôt que d'essayer de trouver des explications satisfaisantes. Dans ce contexte, l'esthétique était simplement une source de «confusions» que les philosophes avaient pour but de clarifier, plutôt que d'édifier de nouvelles théories pour les résoudre. Et les «confusions» esthétiques à l'étude étaient, dans l'esprit des Britanniques de cette époque, celles des anciennes théories des "idéalistes », en particulier celles de Croce, supposément relayé par des passeurs comme Collingwood ou Carritt ${ }^{11}$. Quant aux philosophes moins hostiles à

9. Voir Baxandall, 1991, note 1. Cette influence s'exerce à travers la notion de «re-enactment» (ou "re-production ", comme le veut la traduction) de Collingwood, dans son application à l'interprétation de l'œuvre d'art, dont il ne sera pas possible de parler ici. Cette notion préfigure tout aussi certainement la conception de Richard Wollheim de la critique d'art comme «retrieval» ou «réappropriation». Sur celle-ci, voir Wollheim, 1980, 185-204/169-185. De Baxandall, voir son étude du Baptême du Christ de Piero della Francesca, qui est un exemple parfait de «re-enactment» ou «retrieval» en histoire de l'art, dans Baxandall, 1991, 173-221.

10. Cette idée a aussi été suggérée par John Dewey de manière indépendante dans Art as Experience (1934), quelques années avant Collingwood. Mais, comme nous le verrons, Collingwood proposa une philosophie du langage intéressante pour expliquer cette idée, contrairement à Dewey.

11. Par exemple, les articles réunis dans Aesthetics and Langage introduits comme des «tentatives de diagnostiquer et de clarifier certaines confusions esthétiques» (Elton, 1959,1). Un thème récurrent de ce livre est la «platitude » ou «monotonie » de l'ancienne esthétique «idéaliste». Rétrospectivement, cela s'avère être un commentaire étrange puisque ces articles sont euxmêmes frappants de platitude et de monotonie. 
l'idée même que nous puissions fournir une théorie philosophique, comme Russell, qui était déjà mis à l'écart, ils n'avaient tout simplement rien à dire sur l'esthétique ${ }^{12}$.

De nos jours, l'esthétique analytique est cependant en plein essor, et cette renaissance est due dans une large mesure à la publication en 1968 de Art and its Objects ${ }^{13}$, de Richard Wollheim et de Languages of Art, de Nelson Goodman (Goodman, 1968). Bien que les préoccupations de Wollheim fussent très différentes de celles de ses lointains prédécesseurs «idéalistes » dont les livres n'ont pas été ouverts depuis les années 1940, il chercha néanmoins à les critiquer ${ }^{14}$. L'objectif de la critique de Wollheim est une "théorie", pour laquelle il emploie l'étiquette "théorie de Croce et de Collingwood ${ }^{15}$, et il la décrit en ces termes:

Premièrement, l'œuvre d'art consiste en un état ou une condition intérieure de l'artiste, appelée aussi intuition ou expression; deuxièmement, cet état n'est pas immédiat ou donné; il est le produit d'un processus propre à l'artiste et qui implique articulation, organisation et unification; troisièmement, l'intuition ainsi développée peut être extériorisée sous une forme publique et, en ce cas, nous avons affaire à l'artefact, qui est souvent, quoique cela soit une erreur, pris pour l'œuvre d'art; mais l'intuition peut tout aussi bien ne pas être extériorisée (Wollheim, 1980, 36-37/44).

Le caractère «subjectif » ou «idéel » de l'œuvre d'art en est une caractéristique cruciale:

En effet, selon cette théorie, non seulement un artiste peut créer une œuvre d'art particulière sans jamais effectivement l'extérioriser, mais son aptitude à créer en général des œuvres d'art, ou sa réalisation en tant qu'artiste (pour formuler la chose ainsi), peut se déveloper tout à fait indépendamment d'un quelconque moyen d'extériorisation. L'artiste est artiste uniquement en vertu de sa vie intérieure. [...] on remarquera qu'il est essentiel à la thèse de Croce et de Collingwood que non seulement l'artiste puisse fabriquer des œuvres d'art pour lui-même, mais qu'il puisse être dans une situation telle qu'il ne peut faire des œuvres d'art que pour lui-même: autrement dit, qu'il puisse avoir les intuitions sans avoir socialement aucun moyen de les extérioriser. (Wollheim 1980, 114-115/107-108).

12. Voir Spadoni, 1984.

13. J'utilise ici la seconde édition, augmentée de six essais, Wollheim, 1980. On ne doit pas oublier ici les contributions britanniques à la critique d'art avant Wollheim, par exemple celle d'E. H. Gombrich dans Art and Illusion, publié pour la première fois en 1960 (Gombrich, 1961).

14. Il vaut la peine de noter que, paradoxalement, Wollheim commença sa carrière avec des travaux sur l'idéalisme britannique; voir Wollheim, 1959. En tant que philosophe analytique, Wollheim était plutôt insolite pour cette raison mais aussi à cause de son intérêt pour la psychanalyse. Voir Wollheim, 1974, 1993, 1999.

15. Par exemple, dans Wollheim, 1980, 36/44, 80/80, 114-117/107-110. L'expression «théorie » ou «thèse de Croce et de Collingwood» — c'est ainsi qu'on traduit à tort en français, puisqu'il s'agit au mieux d'un ensemble de thèses - n'est cependant pas de lui, elle était déjà employée dans les années 1950; voir Hospers, 1956, et Donagan, 1958. 
La théorie «idéelle» a donc selon lui trois caractéristiques:

a) l'œuvre d'art consiste en un état ou une condition interne de l'artiste qu'on appelle une intuition ou une expression;

b) Cet état n'est pas donné ou immédiat, il est plutôt le produit d'un processus propre à l'artiste ;

c) Les intuitions ainsi développées peuvent être externalisées dans une forme publique, mais ce n'est pas pour autant nécessaire.

Wollheim s'attaque ensuite à cette théorie à partir des deux points suivants (Wollheim, 1980, 40/46-47). Premièrement, en faisant de l'œuvre d'art quelque chose d' "idéel ", c'est-à-dire d'interne ou de mental, il n'y a aucun objet commun auquel l'artiste et le spectateur peuvent avoir accès :

d) le lien entre l'artiste et le spectateur est rompu.

Deuxièmement, le fait qu'un sculpteur, par exemple, doive tailler un bloc de marbre afin de produire une statue qui soit vue par un spectateur n'est aucunement un aspect mineur ou négligeable de la production d'une œuvre d'art. Autrement dit,

e) la théorie «idéelle» ignore complètement l'importance du médium.

Wollheim a également un autre argument à l'appui de (d), sur lequel je reviendrai au moment approprié ${ }^{16}$. Pour l'instant, il suffit de noter que l'accord avec Wollheim sur ces deux points est aujourd'hui presque universel; je ne les remettrai pas en question. En fait, pris comme objection, (e) n'est même pas vraiment original; cette thèse était discutée dans les années 1930 par Samuel Alexander (Alexander, 1933, 57-59; 1939, 230) et par la suite dans les années 1950 par John Hospers et W. B. Gallie (Hospers, 1956, 293 ; et Gallie, 1959, 18). Le problème des limites intrinsèques du médium

16. Wollheim a aussi discuté de la distinction type/token (Wollheim, 1980, 74/75 sq.) et, dans un article plus tardif, il a raffiné ses critiques de Collingwood au moyen de la distinction entre formes d'art "type" (art type) où il peut y avoir plusieurs instances d'une même œuvre (un poème, une photographie, ou une pièce de musique) et formes d'art "occurrence " (art particular) où cela ne peut pas être le cas (une sculpture ou une peinture), affirmant alors que la conception de Collingwood s'applique au mieux à l'art type (Wollheim 1972). Sur ce point, voir infra, note 39. Une réplique complète dépasserait toutefois le registre du présent article. De plus, la distinction de Collingwood entre l'art et l'artisanat (Collingwood, 1938, 1541) a été critiquée d'un point de vue vaguement "wittgensteinien" comme étant une forme d'essentialisme concernant ce qu'on dit être un «air de famille» ou un concept «ouvert ». (On doit se rappeler ici que cette critique des définitions essentialistes de l'art n'a jamais été présentée par Wittgenstein lui-même, elle trouve son origine dans Weitz, 1956.) Dans Cometti, Morizot, Pouivet, 2000, 14, cet «air de famille » est même censé s'opposer à la «théorie de Croce et de Collingwood ». Qu'il s'agisse ici d'une mécompréhension totale de la riche discussion de Collingwood (il propose six variantes pour une distinction possible et montre qu'elle est poreuse, ne s'engageant donc pas envers une forme d'essentialisme), c'est ce qui est expliqué dans Ridley, 1998. D’autre part, voir Carroll $(1998,49-78)$ pour une discussion critique détaillée. 
avait déjà été soulevé par E. H. Gombrich pour le cas de la peinture dans le premier chapitre de Art and Illusion (Gombrich, 1961) ${ }^{17}$. Selon moi, la critique de Wollheim pose plutôt problème lorsqu'il nomme sa cible la «théorie de Croce et de Collingwood », car il déforme ainsi complètement les conceptions de Collingwood, mais la plupart de ses lecteurs ne le réalisent pas ${ }^{18}$. Aaron Ridley $(1997,1998)^{19}$ a déjà montré que Collingwood n'a pas soutenu la théorie «idéelle », et je ne souhaite pas reproduire ici ses arguments, particulièrement ceux contre l'usage de l'argument (e) contre Collingwood, pour qui le contact physique de l'artiste avec un médium est effectivement une partie essentielle de l'art. En effet, Collingwood a écrit de merveilleuses pages sur Cézanne, Bernard Berenson et Vernon Blake dans lesquelles il affirme clairement l'importance du contact physique lorsque l'artiste travaille avec un médium comme dans les arts visuels (Collingwood, 1938, 144-148), des pages qui contrediraient la thèse selon laquelle (e) s'appliquerait à sa position. Puisque Wollheim ne fournit aucun base textuelle à son affirmation, on peut donc présumer (en prenant par ailleurs appui sur les analyses de Ridley) qu'il est tout simplement coupable d'avoir mal lu Collingwood.

De plus, l'explication de Wollheim ne laisse aucune place à l'affirmation de Collingwood, qui sera cruciale plus loin dans ce texte et qui contredit directement (a), selon laquelle il n'y a rien de tel qu'une émotion préexistant de manière indépendante à son expression/externalisation:

Jusqu'à ce qu'il ait exprimé son émotion, l'homme ne sait pas encore de quelle émotion il s'agit. L'acte d'exprimer l'émotion est ainsi une exploration de ses propres émotions. Il tente de trouver ce que sont ces émotions. Il y a certainement ici un processus orienté: c'est-à-dire un effort orienté vers une certaine fin. Mais la fin n'est pas quelque chose de prévu ou de préconçu, pour laquelle on pourrait penser des moyens appropriés sur la base de notre connaissance de son caractère particulier. L'expression est une activité pour laquelle il ne peut y avoir aucune technique (Collingwood 1938, 111).

[...] l'expression d'une émotion n'est pas, pour ainsi dire, un habit conçu pour convenir à une émotion préexistante: c'est une activité sans laquelle l'expérience

17. On peut même trouver ce point déjà chez Dewey, 1934.

18. Par exemple, tous les clichés possibles sur la "théorie de Croce et de Collingwood" sont ressassés dans Mulhall, 1992, qui semble avoir été écrit sans aucune connaissance de première main des auteurs en question. Sans vouloir trop insister sur ce point dont les exemples se multiplient facilement, je dois également noter qu'on ne retrouve dans Questions d'esthétique par J.-P. Cometti, J. Morizot et R. Pouivet aucune discussion de Collingwood qui fasse montre d'une connaissance détaillée de ses conceptions, bien qu'on trouve ça et là des mentions qui ne vont pas au-delà de — et même assument — la vérité des propositions $(\mathrm{a})-(\mathrm{c})$ de Wollheim, par exemple dans Cometti, Morizot, Pouivet, 2000, 40. Cet ouvrage introductif, par ailleurs excellent, peut induire en erreur sur ce point particulier.

19. Voir également Davies, 2008. Dans Sclafani, 1976, on retrouve une critique plus ancienne de la lecture de Collingwood par Wollheim, mais elle ne semble avoir eu aucun impact. 
de cette émotion ne pourrait exister. Si nous enlevons le langage, nous enlevons du coup ce qui est exprimé (Collingwood, 1938, 244) 20.

On pourrait objecter ici que Collingwood ne fait que se contredire puisqu'il soutient par ailleurs (a) et (c). Après tout, n’a-t-il pas écrit: «[...] un morceau de musique est [...] quelque chose qui pourrait n'exister que dans la tête du musicien" (Collingwood 1938, 151).

Les problèmes «ontologiques» liés au statut de l'œuvre d'art mis à part, ce serait simplement ignorer le fait évident que lorsque Collingwood parle de l' "expression d'une émotion », il parle d'une expression linguistique. Il reproche justement à plusieurs conceptions de son époque l'erreur de concevoir l'expérience esthétique sur le mode de la perception, plutôt que du discours :

La théorie de Croce, quelle que soit la critique qu'on lui adresse, possède à tout le moins le mérite de commencer par la question «comment l'expérience esthétique est-elle possible ? » et d'y répondre en affirmant qu'elle est possible si tout objet esthétique est en quelque sorte un genre de langage et si l'expérience esthétique en général se rapproche davantage de l'acte de parler que de l'acte de percevoir (Collingwood, 1995, 210).

N'importe qui peut composer une pièce musicale exprimant une émotion en se la jouant dans la tête et en la gardant pour soi, mais comment est-ce qu'une pièce musicale peut être jouée "dans la tête» sans aucun recours à un langage musical quelconque? Et, puisque ce qui est exprimé dans un langage est déjà par principe partageable, on peut garder ses pensées pour soi, mais elles sont par principe accessibles et partageables ${ }^{21}$ :

Sans nul doute, un homme peut se parler à lui-même et être son propre auditeur; mais ce qu'il se dit peut en principe être dit à quiconque partage son langage (Collingwood, 1938, 317).

Évidemment, il est clair que le principe guidant la caractérisation et la critique de la «théorie de Croce et de Collingwood» par Wollheim consiste précisément à rejeter cette possibilité; mais non seulement est-il ridicule d'assumer que ce n'est pas ce que Collingwood avait à l'esprit, partant de ce qu'il a écrit (des passages comme ceux-ci ont été ignorés par Wollheim), c'est en plus précisément sur ce point que Bosanquet a critiqué et rejeté les thèses de Croce, un rejet que Collingwood reprendra indépendamment de Bosanquet, en faisant quelques pas de plus en direction d'une théorie plus satisfaisante. L'orientation de mon propos sera ici plus historique, établissant des liens avec Bosanquet, qui rejeta l'esthétique de Croce sur la base du

20. Voir également Collingwood, 1938, 282.

21. Bien sûr, je ne peux défendre ici cette thèse, dont il faut noter qu'elle est clairement d'esprit « wittgensteinien». (Je ne veux certainement pas laisser entendre par là que Collingwood fut influencé par Wittgenstein, car il est fort probable qu'il ne connaissait pas ses idées.) 
fait qu'elle faisait usage d'une conception lamentablement inadéquate du langage et soulignant à la fin de l'article que l'orientation de Collingwood vers une «conception communautaire» de l'œuvre d'art était essentielle à son rejet de la théorie «idéelle».

On retrouve une quantité de preuves selon lesquelles plusieurs philosophes britanniques de la première moitié du $\mathrm{xx}^{\mathrm{e}}$ siècle, comme $\mathrm{E}$. F. Carritt, avaient lu Croce et étaient profondément influencés par ses conceptions. Mais Bosanquet, déjà agé, fit la découverte de Croce seulement vers la fin de sa carrière, et l'Italien n'a pu avoir d'influence durable sur sa pensée. De plus, Bosanquet rejetait fortement à peu près toutes les prémisses de l'esthétique de Croce, comme nous le verrons (Bosanquet, 1920a, 1920b). En ce qui concerne Collingwood, l'idée même d'une «théorie de Croce et de Collingwood" trouve son origine dans la conception exprimée dès 1953 par Carritt, selon laquelle les Principles of Art de Collingwood sont « en grande partie une implémentation de Croce» (Carritt, 1953, 453). Collingwood luimême écrivit à Croce à propos de son livre que «la doctrine qui y est enseignée est pour l'essentiel la vôtre " (Collingwood, 1964, xiv). Il est amplement évident que Collingwood connaissait très bien et appréciait la philosophie de Croce, qu'il découvrit relativement tôt dans sa carrière en raison de son intérêt pour Vico: il traduisit plusieurs livres de Croce, y compris celui sur Vico (Croce, 1913) et la deuxième édition anglaise de son Aesthetics (Croce, 1922) devrait lui être attribuée plutôt qu'à Douglas Ainslie dont le nom apparaît pourtant sur la page couverture ${ }^{22}$. Comme l'a indiqué Alan Donagan, des preuves de cette sorte montrent que ce n'est pas seulement une question de priorité mais aussi une question d'influence (Donagan, 1972, 266). Il est certes clair que Collingwood doit certaines de ses idées à Croce. On le voit bien à l'avant-dernier passage cité (Collingwood, 1995, 210), où Collingwood reconnaît sa dette sur une idée centrale, mais il est clair également qu'il les a considérablement reformulées. En effet, cette même idée, celle du caractère linguistique de l'art, n'a plus le même sens chez Collingwood. Cela soulève donc la question de la portée de cette reformulation: peut-on encore parler d'une "théorie de Croce et de Collingwood», comme on le fait encore aujourd'hui ${ }^{23}$ ? Il se peut que la reformulation de Collingwood soit également une modification de certains points essentiels qui ébranlent la signification même de l'expression "théorie de Croce et de Collingwood». C'est ce que j'ai l'intention de montrer sur un point où, de manière compréhensible, les adversaires de la "théorie de Croce et de Collingwood» ne visent qu'un aspect de celle-ci. Il s'agit de la «théorie idéelle », qu'on retrouve certes chez Croce mais non chez Collingwood; ces adversaires jettent donc le bébé avec l'eau du bain.

22. Les motifs expliquant cette curieuse situation sont exposés dans Knox, 1969, 165.

23. Voir Kemp, 2003. 
On doit se rappeler que, malgré l'admiration qu'il lui vouait, Collingwood ne partageait pas les présuppositions de la philosophie de l'histoire de Croce (encore moins celles de son disciple Giovanni Gentile) et qu'il publia une critique détaillée de celle-ci (Collingwood, 1965, 3-22). Dans ce qui suit, je vais montrer qu'en fait il rejetait aussi certaines présuppositions de l'esthétique de Croce. En outre, mon argument consiste en ceci que sa critique de Croce était déjà pour l'essentiel celle émise par Bosanquet. Tous deux sont d'accord sur le fait qu'ils sont confrontés à une conception erronée de la signification linguistique. Toutefois, et c'est ce que je veux montrer, Collingwood le fit sur la base d'une explication de la signification, qui est des plus intéressantes, là où il n'y en a pas chez Bosanquet. Cela le conduisit à défendre une "conception communautaire» de la signification de l'œuvre d'art qui, incidemment, n'a aucun antécédent chez Croce.

Je montrerai également que Bosanquet et Collingwood ont tous deux tenté d'établir une conception moins «subjective» et du coup plus «objective " que celle de Croce. Il désiraient tous deux mettre l'accent sur le rôle social de l'art. Sur ce point, ils ont écrit des remarques frappantes de similitude. Cependant, comme nous le verrons, leurs affirmations sont similaires seulement de manière superficielle, car elles sont basées sur des prémisses différentes: d'une part, lorsque Bosanquet mentionne ce rôle social de l'art, il ne tente pas de l'expliquer ou, au mieux, ses explications sont basées sur une métaphysique de l'Absolu qui dérive de celle de Bradley. D'autre part, vers la fin de sa vie, Collingwood se distancia de sa première conception idéaliste et commença à développer une philosophie linguistique originale très similaire à celle avancée par le dernier Wittgenstein, qui forme la base de son explication du rôle social de l'art. En bref, si l'art doit jouer un rôle social, sa signification doit être en principe accessible à tous d'une manière qui écarte toute forme forte de scepticisme, et Collingwood soutenait que la signification esthétique est, tout comme la signification dans le langage, déjà sociale ou communautaire. Ces conceptions de Collingwood sont frappantes d'originalité, et je crois qu'elles ont encore une valeur aujourd'hui.

Puisqu'une grande partie de la discussion sur ces questions est constituée d'attributions de thèses à divers auteurs sans aucune base textuelle, et comme je ne peux présumer une connaissance extensive des auteurs concernés de la part de mon lecteur, je vais m'employer dans ce qui suit à citer longuement Croce, Bosanquet et Collingwood lorsque je leur attribue une affirmation, question de rectifier la situation.

\section{Croce et les idéalistes britanniques}

En 1920, Bosanquet publia une analyse de 30 pages de l'esthétique de Croce dans les Proceedings of the British Academy (Bosanquet, 1920a). En philosophe hégélien, Bosanquet appréciait certains aspects de celle-ci, notamment sa capacité à expliquer «le rang de la beauté au sein des expériences de l'esprit » et à rendre justice à la «spiritualité » et à la «simplicité », autrement 
dit: à l'autonomie du beau (Bosanquet, 1920a, 1). Mais s'il ne pouvait pas tout accepter de l'esthétique de Croce, il ne s'agissait toutefois pas d'un rejet total, car il acceptait que l'art et la beauté puissent être des «formes» ou "activités » de l'esprit ${ }^{24}$. Ses principaux arguments s'attaquent à deux affirmations de Croce: premièrement, que l'« intuition » — je réfère ici au concept particulier d'intuizione chez Croce - préexiste à la pensée conceptuelle et, deuxièmement, qu'elle correspond à une phase ou à un niveau distinct de langage. L'Estetica come scienza dell'espressione e linguistica generale de Croce s'ouvre en effet sur une distinction nette entre "intuition" et "concept "; "intuition» étant à ce stade-ci pratiquement synonyme de "perception » (Croce, 1958, 5-6 / 1992, 3). On doit noter qu'il affirme qu'il y a de pures "intuitions " dénuées de concepts: cela montre que la présence d'un contenu conceptuel n'est pas une condition nécessaire, d'où il infère étrangement que, lorsqu'ils sont présents dans des «intuitions ", les concepts

[...] ne sont désormais plus des concepts, car ils ont perdu leur indépendance et leur autonomie. En effet, ils furent un temps des concepts mais il sont maintenant devenus de simples composantes d'intuitions (Croce, 1956, 4 / 1992, 2).

Une intuition est par exemple une «impression de clair de lune, figurée par un peintre» (Croce, 1958, 4 / 1992, 2) ou une «teinte de couleur dans le ciel » (Croce, 1958, 6 / 1992, 5). Ce que Croce voulait dire par le fait que les "intuitions " sont pré-conceptuelles tout en y attribuant éventuellement un contenu conceptuel n'est pas clair, et c'est contre ce point que proteste Bosanquet. De plus, l'«intuition » précède la distinction entre ce qui est réel et ce qui ne l'est pas:

[...] une distinction entre ce qui est réel et ce qui n'est pas réel est extrinsèque à la nature de l'intuition et secondaire (Croce, 1958, 6 / 1992, 3).

Et, non seulement n'y a-t-il pas de distinction sujet-objet dans l' « intuition ", mais le sujet est littéralement en train de générer ou de construire les objets du monde externe à partir des impressions et des sensations:

L'intuition est l'unité indifférenciée de ce qui est perçu et de ce qui est réel et la représentation de ce qui est simplement possible. Dans l'intuition nous ne nous plaçons pas en tant qu'êtres empiriques devant une réalité externe, nous objectivons nos impressions, quelles qu'elles soient (Croce, 1958, 6 / 1992, 4).

Par contre, si nous parlons, à l'instar de certains associationnistes, d'une association qui n'est ni du ressort de la mémoire ni tirée du flux des sensations, mais productrice (formatrice, constructive, permettant de distinguer), alors on peut concéder le point et nier seulement la terminologie. En fait, l'association productrice n'est plus une association au sens où l'entendent ceux qui voudraient

24. En cela, Collingwood est plus près de Croce, comme on peut le voir à même la place accordée à l'art dans Speculum Mentis (Collingwood, 1925). 
tout réduire aux sensations, mais une synthèse, c'est-à-dire une activité spirituelle (Croce, 1958, 10 / 1992, 8).

Ainsi, selon Croce, dans la perception l' « esprit » produit, à travers un processus qui demeure non analysé (à l'exception d'une brève discussion critique de la psychologie associationniste dans Croce, 1958, 9-10 / 1992, 7-8), des "représentations » ou des images d'objets du monde extérieur à partir des stimuli qu'il reçoit. Et cela se produit à un stade précédent - et distinct - de toute activité véritablement conceptuelle de l' "esprit » appelée « logique », en opposition à l' " esthétique» — celles-ci étant les deux activités théoriques de l' "esprit » auxquelles Croce ajoutera plus tard deux activités pratiques. Bien que Croce ne soit pas plus spécifique sur cette question dans ses paragraphes d'introduction, l'activité «conceptuelle » de l' " esprit » consiste en la capacité d'émettre des jugements; la distinction entre ce qui est réel et ce qui ne l'est pas sera établie seulement à ce stade conceptuel.

Cette conception est grevée de certaines difficultés évidentes dont Croce ne discute pas mais qui sont relevées par Bosanquet. À un niveau plus général, Bosanquet ne pouvait assurément suivre Croce. Comme disciple de Bradley, il était partisan d'une forme d'idéalisme «objectif» et non d'idéalisme "subjectif ${ }^{25}$, et la position de Croce lui est fort probablement apparue comme une variante de ce dernier, comme on peut le voir à partir des passages que je viens de citer. S'il y a un stade distinct pour l' "intuition ", il n'y a aucune distinction à ce stade entre ce qui est réel et ce qui ne l'est pas, il n'y a que des images produites par l'esprit. Ainsi, tout commence avec un état purement subjectif (alors qu'il n'y a rien de tel pour Bradley et ses épigones), et il est justifié de se demander s'il fait sens de parler d'une image à un stade où nous ne sommes pas en position de distinguer entre une image et ce que celle-ci dépeint. De manière typique pour un disciple de Bradley, Bosanquet argumente en indiquant une contradiction (ce qui contient une contradiction ne peut exister):

Je crois que la place de l'art et de la beauté au sein des formes de l'esprit est une pure et simple contradiction. Ils précèdent par nature la pensée conceptuelle; c'est le point central de cette gradation. Mais dans les faits, cela est impossible. Il n'y a pas de tel stade précédant la pensée conceptuelle. L'image peut être libérée de tout jugement explicite; mais l'appeler une image, cela signifie qu'on la distingue par la pensée et qu'on se réfère à des conditions objectives. Comment pourrait-elle être autrement une image de quelque chose? Ainsi, l'intuition précède la pensée et en même temps elle est un objet de la pensée [...]. Les exemples de Croce ne laissent planer aucun doute, "les intuitions, ce sont cette rivière, ce lac, etc. » Mais comment cette rivière peut-elle être autre chose qu'un objet de pensée qui a l'identité, la diversité et tout le reste ? (Bosanquet, 1920b, 214).

25. Voir Gandon et Marion dans le présent volume sur cette distinction. 
L'ensemble de cette conception de l'antériorité du l'intuition et du langage sur l'assertion logique et le concept ou la catégorie est un nid de contradictions. On nous affirme que les intuitions sont des choses et les concepts des universaux ou relations. Des choses! On peut donc avoir des intuitions des choses, sans concepts ou catégories, donc de facto sans le travail de l'esprit [...] les pensées de l'identité, de la distinction, de la substance, du tout et de la partie. C’est une absurdité complète (Bosanquet, 1920a, 8).

Mais Croce multiplie ses problèmes en ajoutant ici l'élément le plus distinctif de sa théorie esthétique, à savoir la thèse que l' "intuition » est «expression $»$ :

Tout ce qui est véritablement intuition ou représentation est aussi expression. Tout ce qui n'est pas objectivé dans une expression n'est pas intuition ou représentation, mais sensation ou quelque chose de naturel. L'esprit n'intuitionne que dans la mesure ou il fait, forme, exprime. Celui qui sépare l'intuition de l'expression ne sera jamais en mesure de les rassembler à nouveau. L'acte d'intuitionner ne génère une intuition que dans la mesure où il y a expression. [...] Il est impossible de distinguer entre intuition et expression à l'intérieur de l'acte cognitif (Croce, 1958, 11 / 1992, 8-9).

Intuitionner n'est rien de plus (ni plus, ni moins) qu'exprimer (Croce, 1958, 14 / 1992, 11).

\section{Ce que Bosanquet commente dans ces termes:}

Les deux termes, constamment joints comme descriptifs de la même expérience, véhiculent la conception selon laquelle l'attitude primordiale de l'homme face au monde - avant qu'il ne distingue dans le jugement de perception entre le réel et l'irréel - est celle de l'intuition qui est en même temps, de manière inhérente, une expression imaginative. L'homme ne juge pas: il dépeint, focalise, appréhende dans une image de manière idéale ce qui se présente à son esprit. De manière idéale, ou imaginative - car l'appréhension est idéale ou imaginative lorsqu'elle dépeint et elle n'affirme pas (Bosanquet, 1920a, 2).

On doit ajouter à cela que Croce conçoit cette expression comme étant intrinsèquement esthétique: l'expression fait de chacun de nous un poète, bomo nascitur poeta (Croce, 1958, 18 / 1992, 16). ${ }^{26}$ Cependant, puisque Croce reconnaît qu' "il ne peut y avoir aucune pensée sans mots " (Croce, 1958, 27 / 1992, 25), il doit préserver le stade préexistant à l'intuition de manière indépendante à l'intérieur même du langage:

26. Donc, "chacun de nous est dans une certaine mesure un peintre, un sculpteur, un musicien, un poète, un écrivain" (Croce, 1958, 14, 1992, 11). Collingwood n'en pense pas moins lorsqu'il écrit dans The Principles of Art: "chacun de nos énoncés et chacun de nos gestes est une œuvre d'art » (Collingwood, 1938, 172). Nous verrons cependant que cette thèse n'est pas appuyée sur les mêmes prémisses que celles de Croce. 
La poésie est le langage du sentiment, la prose, celui de l'intellect, mais, puisque l'intellect, dans sa réalité concrète, est aussi sentiment, toute prose possède aussi un côté poétique (Croce, 1958, 30 / 1992, 28).

Ainsi, il y a une dimension poétique à toute expression linguistique, et l'esthétique devient ainsi la science du langage, ou linguistique ${ }^{27}$. Cela rappelle un autre philosophe napolitain, Vico: cette dernière citation est presque une paraphrase de La scienza nuova, Livre I, LIII, $\mathbb{S}$ 218-219 (Vico, 1993, 85). Ce point est crucial dans notre compréhension de la théorie "idéelle» ou de la possibilité même d'une «théorie de Croce et de Collingwood ». En fait, partant des citations vues plus haut, il est clair que Croce a cherché à dégager un stade ou une phase indépendante préexistant à toute activité conceptuelle, où l' «esprit " s'exprime par la production d'images. Même si cette expression est linguistique, et donc conceptuelle, elle conserve une couche esthétique. On se rappellera maintenant la description que Wollheim a faite de la théorie "idéelle" dans les termes de (a)-(c). Laissons (b) de côté pour l'instant et notons que Croce se commet clairement à (a). Il se commet également à $(\mathrm{c})$ :

En fait, les intuitions et expression auxquelles l'esprit donne une forme ne sont pas toutes externalisées et fixées, nos pensées et images ne sont pas toutes exprimées à voix haute, écrites, imprimées, dessinées, colorées, ou exposées en public. On choisit parmi la foule des intuitions formées ou à tout le moins esquissées dans notre esprit. [...] Une intuition ayant été formée, il faut encore se demander si elle mérite d'être communiquée aux autres, et à qui, quand et comment [...] (Croce, 1958, 128 / 1992, 130).

En fait, Croce en a beaucoup à dire sur ce qu'il considère être la traversée du "pons asinorum de l'expression " (Croce, 1958, 144 / 1992, 11), mais il n'est pas nécessaire d'entrer ici dans ces détails. Retenons simplement que Croce se commet à $(\mathrm{c})$. Pour revenir à notre question initiale, si (a) et (c) décrivent l'esthétique de Croce, est-ce qu'elles décrivent celle de Collingwood? Comme je l'ai déjà annoncé, ma réponse est non. On peut voir pourquoi en enquêtant davantage sur la réaction critique de Bosanquet vis-à-vis Croce.

En effet, Bosanquet rejette l'identification entre «intuition » et « expression » proposée par Croce. Cela revient à rejeter la possibilité même d'une phase poétique, distincte et fondamentale, dans le langage. Nous avons vu que Bosanquet avait déjà récusé la possibilité d'une phase distincte pour l' «intuition ", affirmant que l'idée même est contradictoire. Or Bosanquet affirme maintenant qu'il ne pourrait y avoir aucun langage sans aspect «conceptuel » :

27. Sur l'identification de l'art au langage, voir de Mauro, 1969, chapitre IV. Cependant, j'aimerais ici me distancer de toute discussion concernant l'interprétation juste de la philosophie du langage de Croce et de sa valeur pour la linguistique. Bien que je doute qu'il y en ait une, il est intéressant de noter avec Tullio de Mauro qu'il y avait une tradition crocéenne dans la linguistique italienne, avec Giovanni Gentile et ses étudiants. 
Communiquer à un esprit ce qu'il ne sait pas dans les termes de ce qu'il sait déjà - ce qui est la fonction primaire du langage - cela ne pourrait aucunement être réalisé. L'esprit primitif, loin d'être purement contemplatif et imaginatif, est immergé dans la pratique; et à chaque moment il doit exiger de communiquer au moyen d'éléments du discours qui sont définis et séparables. «Traverse deux rivières et tourne en amont à la troisième ». Comment la vie primitive pourrait-elle se poursuivre sans une communication de ce genre? Et comment une phrase de ce genre pourrait-elle transmettre un conseil utile si les points sur lesquels une erreur est possible n'étaient pas des références distinctes et reconnaissables? [...] C'est là une contradiction extraordinaire que d'en appeler au contraste déjà remarqué par Aristote entre l'assertion ou la proposition logique et la phrase qui énonce un souhait ou un ordre, comme l'a fait Croce à plusieurs reprises. Il considère que seul le premier terme du contraste a une signification en fonction d'un accord, alors que le second serait une sorte de poésie primitive qu'il croit dénuée d'une telle signification et qui la précéderait même. Mais les deux types de phrases ont de la même manière une signification "conventionnelle» ou logique, bien qu'une d'entre elles ne soit pas assertive, et c'était pour enfoncer ce clou que j'ai employé un impératif dans mon exemple. Bref, le langage n'est pas ce qu'il est sans son côté conceptuel, et faire correspondre le langage à l'intuition, pour ensuite traiter l'intuition comme précédant la pensée, c'est briser et renverser toute la conception de l'unité de l'esprit humain (Bosanquet, 1920a, 8-9).

On doit noter ici que Bosanquet se base dans ce passage sur une conception rudimentaire du langage comme moyen de communication, c'est-àdire comme vébicule des pensées. Il ne discute pas davantage cette conception mais la considère certainement moins absurde. Je vais revenir sur ce point. De plus, Bosanquet croit qu'avec le langage, dont la fonction est réduite à communiquer, il n'est tout simplement pas possible de parler de «beauté »:

Or, lorsque le langage est dépouillé de son plein potentiel de signification afin de le faire correspondre ou de l'aplanir avec l'expression en général, la manœuvre est auto-destructrice. Le langage devient alors un geste ou un air, et comparer un air ou un geste au langage, c'est alors comparer une chose à ellemême. Si l'expression, la beauté et le langage sont considérés en principe comme précédant la pensée et la signification explicite, le problème de la beauté est alors traité comme s'il était déjà réglé, alors qu'en vérité, il n'a même pas été soulevé. La beauté n'est pas un donné, c'est une transformation; ce n'est pas un don que l'on reçoit à la naissance, c'est la réalisation d'un adulte (Bosanquet, 1920a, 6).

Dans le contexte du présent article, cela est d'autant plus intéressant qu'il médite sur le fait que la signification de l'œuvre d'art puisse être réduite à ce qui revient à être une image "privée». En d'autres termes, Bosanquet souligne le fait que Croce ignore le rôle de l'artéfact ou du médium en art:

Nous avons dit que Croce rejettait la réalité du monde extérieur. Avec ce rejet, la singularité de l'intuition-expression est vigoureusement intensifiée. Comme on le soutient habituellement, il y a dans l'art un intérieur et un extérieur, en un certain sens. Certes, la beauté réside dans l'imagination et non pas dans le 
caractère physique, mais les efforts en vue d'une expression semblent être des efforts en vue de donner une réalité extérieure à quelque chose d'intérieur. À un tel sentiment de l'intérieur et de l'extérieur, Croce ne fait aucune concession. Le monde extérieur n'est aucunement réel, l'art est la plus réelle des choses. Mais alors, comment l'art peut-il faire partie du monde extérieur ou être dans une quelconque connexion avec des processus ou intermédiaires physiques? (Bosanquet, 1920a, 10).

On doit mettre l'accent sur ce point, et c'est pourquoi je cite un passage de ses Three Lectures on Aesthetics:

[Croce] oublie [...] que, bien que le sentiment soit nécessaire à son incarnation [embodiment], l'incarnation est également nécessaire au sentiment. Dire que c'est parce que la beauté implique un esprit qu'elle est un état intérieur et que son incarnation physique est quelque chose de secondaire et d'accessoire n'ayant lieu que pour assurer la permanence et la communication - cela me semble être une profonde erreur de principe, un faux idéalisme (Bosanquet, 1915, 67-68).

Dans ces dernières citations, Bosanquet rejette en effet (c) en employant un argument qui préfigure l'usage de (e) par Wollheim. Pour cette raison, il me semble inapproprié d'attribuer à Bosanquet la théorie «idéelle». Je ne veux pas dire par là que je trouve ses conceptions acceptables, mais plutôt qu'il serait précipité d'étiqueter ainsi ses conceptions sans jeter un coup d'œil à ce qu'il a écrit en réalité.

Alexander avait déjà noté le défaut fondamental de la critique de Bosanquet : ce qu'il dit peut certes être vrai, mais il ne nous dit pas pourquoi (Alexander, 1939, 220). En effet, Bosanquet ne justifie pas que l'incarnation dans un artéfact soit nécessaire à l'expression artistique du sentiment ${ }^{28}$. Comme je l'ai indiqué, Bosanquet est dans sa critique parti d'un modèle du langage selon lequel, pour le dire approximativement, celui qui parle forme une intention et l'exprime ensuite dans le langage en employant une signification conventionnelle, alors que l'auditeur déchiffre la signification conventionnelle des mots employés de façon à se former une image de l'intention de celui qui parle. Selon Bosanquet, la notion crocéenne d' "intuition" ne cadre pas dans ce modèle. Wollheim va dans le même sens lorsqu'il bousille son interprétation de Croce (oublions un moment l'occurrence de "Collingwood» dans l'expression «théorie de Croce et de Collingwood»), en affirmant que Croce distingue l'art du langage: dans le cas de l'art, dit-il, l'artiste

[...] peut être dans une situation où il peut produire des œuvres d'art pour lui seul, autrement dit, il est possible qu'il ait les intuitions et qu'il n'y ait aucune manière de les externaliser dans la société (Wollheim, 1980, 115/108).

Wollheim met alors ce point en contraste avec le cas du langage : lorsqu'on forme des pensées dans le médium du langage, il est vrai que l'on peut

28. La critique de Croce par Alexander est en effet analogue de celle de Bosanquet en ceci qu'il relève aussi les défauts de la notion d'intuition, comme le fait qu'elle soit antérieure au langage et purement privée (Alexander, 1933, 132-134, 1939, 219-220). 
s'empêcher de les partager ou de les «employer de manière externe ", mais il est toujours possible de le faire en principe. La théorie n'est donc pas à propos du "penseur ayant un médium de pensée qu'il utilise seulement pour lui-même ", mais plutôt à propos du "penseur qui n'a aucun médium de pensée » (Wollheim, 1980, 116/109). Cela ne rend clairement pas justice à Croce, qui identifie art et langage. Comme nous l'avons vu dans notre dernière citation de celui-ci, toute intuition formée est en principe employable de manière externe, pour utiliser le jargon de Wollheim. En fait, cela contraint même Croce à affirmer que la différence entre Raphaël et un peintre incompétent quelconque ne réside pas dans la capacité à reproduire sa propre «intuition» sur un canevas, mais dans la qualité de l'«intuition » ellemême (Croce, 1958, 12-13/ 1992, 9-10). (Comme le montre ce dernier point, la théorie de Croce n'est pas sans graves difficultés.)

Or, si on applique au cas de l'art la conception du langage présentée ici de manière rudimentaire, l'affirmation de Bosanquet revient à ceci (évidemment dans des mots qui ne sont pas les siens): Croce pense qu'il y a quelque chose de tel qu'une intention-dans-la-tête-de-l'artiste, son externalisation (contingente) dans un objet physique, et la réplique-de-l'intention-del'artiste-dans-la-tête-du-spectateur, formée en contact avec l'objet physique; mais cela ne pourrait être le cas s'il n'y avait pas de façon préliminaire un ensemble de conventions, comme c'est le cas pour la communication dans le langage selon la conception présentée plus haut, pour l'externalisation de l'intention-dans-la-tête-de-l'artiste dans un objet physique, conventions qui sont partagées par les spectateurs qui sont alors en position de former dans leurs têtes des répliques-de-l'intention-de-l'artiste. Ainsi, Croce ne peut expliquer comment nous pourrions communiquer à travers l'œuvre d'art. Ce dernier point est important pour Bosanquet, car il affirme dans ses Three Lectures on Aesthetics que

[...] l'attitude esthétique est celle où nous avons un sentiment qui est incarné dans un objet à un point tel qu'il restera fixe pour être observé et, en principe, pour être observé par quiconque (Bosanquet, 1915, 6).

De plus, Bosanquet croyait qu'il est essentiel au sentiment esthétique d'être un "sentiment commun". Ainsi, il affirme que "vous pouvez faire appel aux autres afin qu'ils le partagent; et sa valeur n'est pas diminuée par le fait d'être partagée " (Bosanquet, 1915, 5). Les raisons poussant Bosanquet à faire appel à un "sentiment commun" sont plutôt complexes; elles ont quelque chose à voir avec sa croyance selon laquelle l'art mène à une expansion du soi, à la fois de l'artiste et du spectateur ${ }^{29}$. Si l'art devait jouer un tel

29. À son tour, cela est également fondé dans sa métaphysique de l'Absolu comme une complétude infinie et une fusion des esprits finis, mais il n'y a ici aucune nécessité à développer ce point. Voir Lang, 1968, 380-382. Il est certain que cet aspect «hégélien » de la philosophie de Bosanquet est dépassé depuis un bon moment. Il n'y a aucun appel équivalent à un «absolu» chez Collingwood. 
rôle dans le développement du caractère ou de la conscience, il serait alors nécessaire d'en appeler à des "sentiments communs ". Nous retrouvons ici les motivations sociales derrière l'esthétique de Bosanquet, et il n'est pas nécessaire de les expliciter davantage. Comme nous le verrons, la théorie de Collingwood était également motivée par le rôle social qu'il impute à l'art.

On devrait toutefois noter que Bosanquet n'est pas clair sur ce qu'il veut dire par l'«incarnation» des «sentiments» dans l'œuvre d'art, et qu'il n'a manifestement senti aucune nécessité de justifier le modèle du langage sur lequel il se base ici. Ce modèle n'est pas dépourvu de difficultés. Par exemple, cette imagerie ouvre la porte naturellement au scepticisme, puisqu'il n'y a aucune garantie que les répliques-de-l'intention-de-l'artiste-dans-la-têtedes-spectateurs correspondent à l'intention-dans-la-tête-de-l'artiste, car il n'y a aucun accès direct à celle-ci, mais uniquement un accès par le biais de son encodage, pour ainsi dire, dans l'objet physique. Tout cas de malentendu, et il ne manque pas de tels cas en art, semble alors confirmer la véracité de ce scepticisme, auquel font aussi face les théories « intentionnalistes » contemporaines telles que celles de Wollheim ou de Levinson ${ }^{30}$. De toute façon, le problème de la position de Bosanquet est lié au fait qu'il n'a pas expliqué comment il se fait que l'émotion ou le sentiment esthétique puisse être partagé, puisqu'il fait usage dans sa critique de Croce de la conception du langage selon laquelle les «significations» demeurent essentiellement privées. Je crois que Collingwood a conçu son esthétique - une théorie qu'on a récemment appelée, avec raison, une théorie de la "performance » ${ }^{31}$ - précisément dans le but d'éviter cet écueil (qu'il réussisse à l'éviter en bout de ligne, c'est une autre histoire).

Dans The Principles of Art, Collingwood ne discute nulle part ouvertement de l'esthétique de Croce, et nous ne pouvons pas voir clairement ce qu'il en a tiré et ce qu'il en a rejeté. Toutefois, de ce que je comprends de Collingwood, sa théorie esthétique s'accorde à la critique de Croce par Bosanquet. Même son ancien maître, Carritt, qui s'était amouraché de Croce, a indiqué que le concept d'expression de l'Italien n'est «pas la communication » et qu'avec celui-ci nous demeurerions "confinés à nous mêmes » (Carritt, 1932, 90-91). Nous ne serions alors pas en mesure d'expliquer comment l'artiste et son spectateur peuvent partager quoi que ce soit par le biais de l'œuvre d'art - une inquiétude qui n'a rien de "post-moderne». Collingwood était très conscient de cela et s'est exprimé très clairement dans The Principles of Art:

30. Voir Wollheim, 1987, ainsi que les articles réunis dans Levinson, 1996 et 2006. C'est bien sûr une thèse audacieuse lorsqu'elle n'est appuyée par aucun argument, mais une discussion détaillée nous mènerait ici trop loin.

31. Voir Davies, 2008, 168. Bien entendu, la théorie de la «performance » de l'œuvre d'art est controversée, comme n'importe quelle autre théorie, mais on ne peut dire qu'elle appartient à une ère «idéaliste » révolue. Pour une défense récente, voir Davies, 2004. 
Nous supposons que l'expérience esthétique en soi est, dans les deux cas, une expérience purement intérieure, qui prend entièrement place dans l'esprit de la personne qui a cette expérience. Mais cette expérience intérieure est censée être dans une double relation à quelque chose d'externe ou de corporel. (a) Pour l'artiste, l'expérience intérieure peut être extériorisée ou convertie en un objet perceptible, bien qu'il n'ait aucune raison intrinsèque pour qu'il en soit ainsi. (b) Pour l'audience, il y a un processus converse: l'expérience extérieure vient en premier et elle est convertie en cette expérience intérieure qui est, elle seule, une expérience esthétique. [...] Si l'expérience esthétique, du côté de l'artiste, est quelque chose de complètement indépendant de telles choses extérieures, mais qu'il y a quelque chose du côté de l'audience qui dépend de ces choses extérieures et qui sont dérivées de la contemplation de celles-ci, comment est-ce une expérience du même genre dans les deux cas, et comment peut-il y avoir quelque communication que ce soit? (Collingwood, 1938, 301-302).

Dans le cas de la peinture discuté ici, sa propre conception à ce sujet était au contraire que l'expérience du spectateur est «identique à celle du peintre»(Collingwood, 1938, 308). Très clairement, les lecteurs anglais de Croce étaient mal à l'aise avec certains aspects de sa théorie esthétique et n'y ont pas adhéré en bloc, sans nuances. Cela a toutefois pour effet qu'il est alors nécessaire de fournir les fondements théoriques nécessaires à la possibilité du partage de la signification dans l'œuvre d'art. Tout le deuxième livre de Principles of Art est donc consacré à l'imagination, ce qui semble plutôt crocéen, mais Collingwood y développe une philosophie du langage beaucoup plus intéressante que le modèle du langage auquel Bosanquet fait appel dans ses critiques de Croce, et qu'il améliorera plus tard dans son dernier ouvrage, The New Leviathan, lequel n'est assurément pas crocéen. Autrement dit, la base de la théorie de Collingwood ne vient pas de chez Croce (ou de Bosanquet), et la différence est, là encore, une différence essentielle puisqu'elle mène au rejet de la théorie «idéelle» esquissée en $(\mathrm{a})$ - (c). Ce qui suit implique une reconstruction de la pensée de Collingwood sur ces problèmes, qui évoluait encore jusqu'à sa mort précoce à l'âge de cinquantetrois ans en 1943. Il faut donc éviter de chercher dans des ouvrages plus anciens comme The Idea of History (Collingwood, 1994), pratiquement terminé en 1936, ou même dans The Principles of Art, terminé en 1938, où elle est en gestation; malheureusement, je devrai passer sous silence bien des problèmes d'interprétation ${ }^{32}$.

\section{L'esthétique de Collingwood}

La meilleure façon de comprendre la conception du langage et de l'art du dernier Collingwood est peut-être de la contextualiser, c'est-à-dire de la

32. Il y a en effet plusieurs problèmes soulevés ici par les spécialistes et sur lesquels je ne me prononcerai pas. Par exemple, Alan Donagan voyait dans The New Leviathan un « recul vers l'associationnisme » (Donagan, 1962, 54-56) là où j'affirme plus loin que Collingwood est allé au-delà de la psychologie vers un point de vue complètement linguistique. 
comprendre dans le contexte plus large dans lequel elle s'insère, en la contrastant avec la théorie de laquelle cette conception s'est émancipée. Ma compréhension globale de l'évolution de la philosophie de Collingwood est qu'après avoir été proche du "réalisme d'Oxford » de ses professeurs avant la Première Guerre mondiale, il devint un «idéaliste » plutôt orthodoxe, pour ensuite se départir vers la fin des années 1930 d'une grande partie de cet héritage "idéaliste", s'orientant vers des conceptions plus matures (qu'il n'acheva jamais complètement jusqu'à sa mort précoce) $)^{33}$. Ses livres Speculum Mentis (Collingwood, 1924) et An Essay on Philosophical Method (Collingwood, 2005) fournissent la meilleure expression de son «idéalisme». La nature précise de ce dernier est matière à discussion, mais il est clair qu'elle est d'une forme hautement originale. Par exemple, sa «dialectique de l'esprit» comme une «échelle des formes » dans Speculum Mentis était un système ambitieux allant du pur «flux » des sensations à l'action rationnelle, et dont les stades intégraient l'art, la religion, la science, la philosophie et l'histoire. On pourrait reproduire une version simplifiée de son aspect «psychologique» de la manière suivante ${ }^{34}$ :

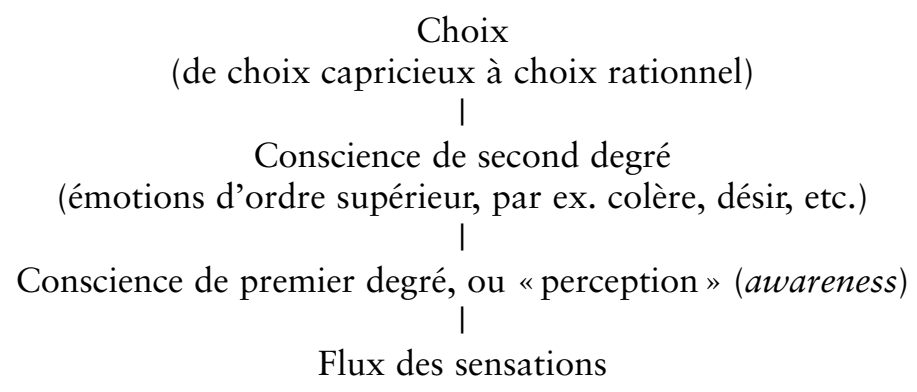

Brièvement: on peut dire qu'en partant du «flux» des sensations, l'agent prend connaissance de son entourage et de sa propre réaction émotionnelle à celui-ci, et qu'à son tour il peut développer ses propres désirs et émotions d'ordre supérieur (ou «émotions de l'intellect », comme le dit Collingwood), par exemple: être choqué d'avoir eu honte. Les désirs se présentant comme des buts à réaliser, vient alors le moment de décider lesquel sera poursuivi, étant donné la compréhension personnelle de sa propre situation par l'agent. Les diverses activités théoriques de l'être humain nous aident à passer d'un niveau à l'autre. Ainsi, par exemple, The New Leviathan contient une longue discussion de l'usage de la pensée philosophique et historique dans le choix du «devoir ", parmi les possibilités d'action offertes, c'est-à-dire de l'action rationnelle par excellence (Collingwood, 1992, chapitres XIII à XVIII).

33. Pour un compte rendu de la main de Collingwood sur sa propre évolution, voir son Autobiography (Collingwood, 1939).

34. Pour un diagramme plus complet, voir Mink, 1969, 117. 
Dans ce cadre général, la place initiale de l'art se situe au niveau de la conscience de premier degré ou "perception ", où l' «imagination » est nécessaire pour l' 'expression» par l'agent de ses propres sentiments concernant les événements qu'il vit. L'idée semble être que l'agent en vient à "percevoir» en portant «attention" aux parties du flux «total» de ses sensations, mais que ces sensations sont toujours accompagnées d'une charge émotionnelle ${ }^{35}$, celle-ci pouvant toutefois être trop douloureuse ou lourde de conséquences pour que l'agent l'accepte et la reconnaisse complètement comme la sienne, tendant ainsi à la désavouer. Le processus menant à percevoir notre propre réaction émotionnelle face à des événements de notre vie peut ainsi échouer et mener à une étrange situation dans laquelle nous ressentons certaines émotions tout en les désavouant, c'est-à-dire en refusant de reconnaître que nous ressentons ce que ressentons. Collingwood appelle cette situation une "corruption de la conscience". Il la décrit en employant presque les termes de Hume sur le passage des impressions sensibles aux idées:

Nous dirigeons d'abord notre attention vers un certain sentiment [feeling] ou nous en prenons conscience. Ensuite, nous prenons peur à ce que nous avons reconnu: non pas parce que le sentiment, comme impression, est une impression alarmante, mais parce que l'idée dans laquelle nous convertissons ce sentiment s'avère être une idée alarmante. Nous ne pouvons voir comment la dominer et évitons de persévérer dans nos tentatives de le faire, donc nous abandonnons l'idée et nous nous tournons vers quelque chose de moins intimidant... C'est ce que j'appelle la «corruption » de la conscience; car la conscience s'autorise à être soudoyée ou corrompue dans l'exécution de ses tâches, se laissant distraire d'une tâche formidable au profit d'une tâche plus facile (Collingwood, 1938, 217).

Collingwood considérait la «corruption de la conscience» comme malsaine et potentiellement dangereuse :

À moins que la conscience fasse son travail avec succès, les faits qu'elle offre à l'intellect, les seules choses sur lesquelles l'intellect peut construire son tissu de pensées, sont faux depuis le début. Une conscience honnête donne à l'intellect une fondation solide sur laquelle construire: une conscience corrompue force l'intellect à construire sur des sables mouvants. Les faussetés qu'une

35. Sur l'attention, voir Collingwood, 1938, 203sq., Collingwood, 1992, 4.5-4.6, et, sur les sensations et leur charge émotionnelle, voir Collingwood, 1938, 232; Collingwood, 1992, 4.1 et 4.62. Ces remarques appartiennent à proprement parler à la sphère de la psychologie. Malheureusement, Collingwood ne cite aucune source, mais ce n'est manifestement pas de la fabulation. Par exemple, la notion d' "attention » et l'idée que les sensations sont toujours accompagnées d'une charge émotionnelle a été introduite par James Ward dans la psycho-

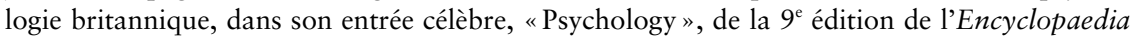
Britannica (Ward 1886), et on la retrouve, avec d'autres idées sur les émotions d'ordre supérieur, dans ses Psychological Principles, rédigés en fin de carrière (Ward, 1918). Il y a aussi de nombreuses références à William James chez Collingwood, par exemple un rejet de sa théorie des émotions dans Collingwood, 1938, 232-233. 
conscience malhonnête impose à l'intellect sont des faussetés que l'intellect ne peut jamais corriger. Et dès que la conscience est corrompue, les sources mêmes de la vérité sont empoisonnées. L'intellect ne peut rien construire de solide. Les idéaux moraux sont des châteaux suspendus dans les airs. Les systèmes politiques et économiques sont de simples toiles d'araignées. Même la santé commune et la santé physique ne sont plus sûrs. [...] De la même manière que l'existence même de la vie d'une communauté dépend d'un commerce honnête entre les hommes - la tutelle de cette honnêteté étant acquise non pas dans une classe ou dans une section, mais chez tout un chacun, l'effort en vue d'une expression des émotions, l'effort de dépasser la corruption de la conscience, est un effort qui doit être fait non pas uniquement par des spécialistes, mais par quiconque utilise le langage et à chaque fois qu'il l'utilise. Chacun de nos énoncés et chacun de nos gestes est une œuvre d'art. Il est important pour chacun de nous que dans la réalisation de l'œuvre nous ne nous trompions pas, peu importe que nous trompions les autres. Celui qui se trompe lui-même fait germer en lui un grain qui, s'il ne le déracine pas, évoluera vers une sorte quelconque de malice, de maladie mentale, dans une sorte de bêtise, de sottise et de folie. Le mauvais art, la conscience corrompue, est la véritable radix malorum. (Collingwood, 1938, 284-285).

(Comme je l'ai déjà dit, je reviendrai sur la dimension sociale un peu plus loin.) Ainsi, on doit reconnaître ses propres émotions pour pouvoir y faire face et éviter des conséquences désastreuses. Peut-être que l'Adolphe de Benjamin Constant aurait pu servir ici d'illustration littéraire aux idées de Collingwood, bien que ce dernier n'en fasse aucune mention: ses références sont celles que l'on rencontre habituellement, c'est-à-dire Spinoza et Freud ${ }^{36}$. Toutefois, il a probablement repris l'idée de Croce, qui écrivait:

En travaillant sur ses sensations, l'homme se libère. En les objectivant, on s'en détache et on les domine. La fonction libératrice et purificatrice de l'art est un autre aspect de son caractère d'activité. L'activité libère précisément parce qu'elle chasse la passivité (Croce, 1958, 24/ 1992, 22).

La distinction entre l'activité et la passivité est déjà ici une référence à Spinoza sur actio et passio, comme l'expose clairement Collingwood:

36. Il y a quelques traces précieuses de la psychanalyse dans le développement de Collingwood. Herbert Read avait toutefois déjà intégré les idées de Freud dans Art and Society publié en 1936 (Read, 1966, 82-95), et il est probable que Collingwood l'avait lu. Bien enten$\mathrm{du}$, il avait une connaissance de première main de la psychanalyse: respectant son principe selon lequel on ne peut parler d'une activité sans l'avoir soi-même pratiquée, il s'est livré à une psychanalyse complète. (Il était dessinateur (ses reproductions d'inscriptions latines sur pierres dans les îles britanniques, qui furent sa contribution au projet de Theodor Mommsen d'un Corpus Inscriptionum Latinarum ont été publiés après sa mort ) et il fut pianiste également: il pensait donc pour ces raisons qu'il pouvait parler d'art en connaissance de cause. En ce qui concerne sa philosophie de l'histoire, il était aussi l'archéologue et l'historien le plus éminent de la Grande-Bretagne à l'époque romaine de sa génération.) Son collègue John Mabbott a commenté sarcastiquement les «cinquante pleines sessions» de psychanalyse de Collingwood: «je crois qu'elles lui ont causé de sérieux torts» (Mabbott, 1976, 76). 
Cette corruption de la conscience a déjà été décrite par les psychologues de leur propre manière. Ils appellent répression le déni des expériences : par projection, ils décrivent l'attribution de celles-ci à d'autres personnes: par dissociation, ils décrivent la consolidation de celles-ci dans une masse d'expérience homogène en elle-même (comme cela peut bien être le cas si le déni est systématique): et ils qualifient de construction fantaisiste la construction d'une expérience expurgée que nous admettrons être la nôtre. Ils ont aussi montré l'effet désastreux qu'ont ces corruptions de la conscience sur la personne qui en souffre, lorsqu'elles deviennent habituelles. Spinoza nous a enseigné la même leçon il y a longtemps, lui qui a exposé mieux que quiconque la conception de la conscience honnête et son importance comme fondement d'une vie mentale saine. Pour lui, le problème de l'éthique est la question de savoir comment l'homme, mû par ses sentiments, peut les incorporer de façon telle que sa vie puisse devenir, de passio continue, subissant les choses, une actio consciente, une action sur les choses. La réponse qu'il donne est étonnamment simple. [...] Dès que nous formons une idée claire et distincte d'une passion, celle-ci cesse d'être une passion (Collingwood, 1938, 218-219).

Pour Collingwood, le rôle de l'art est d'aider à combattre la «corruption de la conscience » au moyen de l'expression imaginative de nos propres émotions $^{37}$. Je ne peux ici faire mieux que de citer le magnifique paragraphe de conclusion de Principles of Art:

[L'art] doit être prophétique. L'artiste doit prophétiser non pas au sens de prédire les choses qui vont arriver, mais au sens de dire aux membres de son audience, au risque de leur déplaire, le secret de leur propre cœur. Comme artiste, son travail consiste à révéler, à confesser. Mais ce qu'il doit énoncer, ce ne sont pas ses propres secrets, comme la théorie individualiste de l'art veut nous le faire penser. Comme porte-parole de sa communauté, les secrets qu'il doit énoncer sont ceux de sa communauté. La raison pour laquelle celle-ci a besoin de lui est qu'aucune communauté ne connaît son propre cœur; et sans cette connaissance, une communauté se trompe à un point tel que l'ignorance signifie la mort. Contre les maux qui proviennent de cette ignorance, le poète comme prophète ne propose aucun remède, car il en a déjà donné un. Le remède, c'est le poème lui-même. L'art est la médecine de la communauté contre la pire maladie de l'esprit, la corruption de la conscience (Collingwood, 1938, 336).

Je reviendrai plus loin, dans les remarques finales, sur la conception de Collingwood du rôle social de l'artiste, une conception qui préfigure très bien les conceptions mieux connues d'Iris Murdoch dans The Sovereignty of Good over Concepts (Murdoch, 1967) ${ }^{38}$. Pour l'instant, j'aimerais souligner que Collingwood ne pourrait pas être dans la position de nous dire que l'art

37. Bien entendu, nous ne sommes pas loin de la «catharsis", et Collingwood en était conscient; voir Collingwood 1938, 110.

38. Voir Davis, 2008, 173. Par ailleurs, il importe de mentionner les connexions avec les conceptions d'Herbert Read dans son livre de 1936, Art and Society; voir par exemple, Read, 1966, 95. 
peut jouer un rôle social fondamental, s'il n'avait pas une explication crédible de la possibilité, pour les membres d'une communauté, de partager des émotions au moyen de l'art; cette thèse sur le rôle social de l'art souligne donc la remarque que j'ai faite concernant la nécessité de fournir une explication de l'art montrant comment l'artiste et le spectateur partagent quelque chose à travers l'œuvre d'art. Telle est précisément la tâche principale des livres II et III des Principles of Art de Collingwood, que personne ne lit de nos jours, tant est tenue pour acquise la critique que Wollheim a faites de la «théorie de Croce et de Collingwood», dont la seule base textuelle se trouve dans le livre $\mathrm{I}^{39}$.

39. En fait, dans le livre I, Collingwood a écrit des remarques qui donnent des munitions à la lecture erronée de Wollheim, par exemple, lorsqu'il écrit qu' « une œuvre d'art peut être complètement créée si elle a été créée comme une chose qui ne se situe que dans l'esprit de l'artiste" (Collingwood, 1938, 134). Mais Wollheim ne réalise manifestement pas que Collingwood visait à installer ses arguments à l'intérieur d'un moule «thèse-antithèse-synthèse » typique des idéalistes britanniques, et que, par conséquent, sa conception véritable n'est exprimée adéquatement que dans le livre III, une fois les défauts des conceptions exprimées au livre I corrigés par les considérations que l'on trouve dans le livre II. Collingwood est plutôt explicite sur ce point à plusieurs endroits dans le livre, et également à la fin du livre I, où il souligne que la théorie du livre III résulte de l' «union» des lignes de recherche des livres précédents (Collingwood, 1938, 153) ainsi que dans une note de bas de page révélatrice tirée de la section dont cette dernière citation a été tirée: "Le lecteur comprendra, je l'espère, que tout ce que j'ai dit dans le livre I est volontairement provisoire et que ma théorie de l'art n'est pas exposée avant le livre III" (Collingwood, 1938, 136). Wollheim fut probablement mis au courant de cette note de quelque manière, car, quelques années après son livre, il publia un article au titre révélateur: "On an Alleged Inconsistency in Collingwood's Aesthetic» (Collingwood, 1972). Dans cet article, Wollheim refuse tout simplement de reconnaître la nature «dialectique » de l'argument de Collingwood et insiste pour que l'on prenne ses remarques au pied de la lettre, affirmant ainsi avoir trouvé une incohérence chez Collingwood. Wollheim affirme également que «la conception avancée par Collingwood dans le livre III de Principles of Art est très fragmentaire" (Wollheim, 1972, 77), question de l'écarter et d'insister sur la thèse qu'il n'y a que les conceptions exprimées dans le livre I qui peuvent être affirmées comme telles. Au bas mot, c'est de l'hypocrisie exégétique. Comme je l'ai déjà souligné dans la note 16 plus haut, c'est dans le même article que Wollheim introduit une distinction entre art "type » et art "occurrence ", afin d'appuyer la thèse que la philosophie de Collingwood s'applique tout au plus au cas de l'art «type ». Comme je l'ai dit dans cette note, je ne discuterai pas de ce point, mais on doit noter que la citation ci-dessus est juste si l'on a à l'esprit un art "type " tel que la musique et qu'en effet Collingwood n'énonce précisément cette thèse qu'en référence à la musique et à la poésie dans Collingwood, 1938, 132 et 151. Hélas, ses propos sont à l'occasion confus, comme au moment où il confond images, pièces musicales et poèmes dans Collingwood, 1938, 134) De toute façon, il est vrai que cette conception ne se transpose pas à l'art "occurrence " comme la peinture, mais la discussion de la peinture par Collingwood dans Collingwood, 1938, 144-148 ne laisse aucune place pour la "théorie idéelle » non plus, comme je l'ai déjà mentionné. Il n'est pas nécessaire de feindre le désespoir, comme le fait Wollheim, mais plutôt de faire l'effort de comprendre la conception qu'il rejette si rapidement. Une lecture inadéquate et injuste demeure une lecture inadéquate et injuste, et le moins que l'on puisse faire est d'essayer de comprendre ce que Collingwood voulait vraiment dire. 
On doit noter également que Collingwood n'a pas défini l'art comme étant l' "expression d'une émotion" en tant que simple "activité" (par opposition à un état), mais en tant qu'activité "cognitive », c'est à dire:

[...] une activité par laquelle nous prenons conscience de nos propres émotions (Collingwood 1938, 292).

En théorie, l'artiste est une personne qui en vient à se connaître lui-même, à connaître ses propres émotions (Collingwood, 1938, 291).

L'activité qui génère une expérience artistique est l'activité de la conscience. Cela exclut toutes les théories de l'art qui situent son origine dans la sensation ou dans les émotions (Collingwood, 1938, 273) ${ }^{40}$.

C'est là une différence cruciale qui est souvent ignorée par les critiques hâtifs ${ }^{41}$. Incidemment, la dernière citation est un rejet implicite de Croce. On doit se rappeler encore une fois le système général dans lequel est implantée la philosophie de l'art de Collingwood: cette implantation définit l'intérêt premier de l'art comme étant cognitif ${ }^{42}$.

Revenons maintenant au système d'ensemble de Collingwood. Malgré la volonté de concevoir une échelle des diverses activités de l'être humain (art, religion, philosophie, etc.), il n'est question ici d'une orthodoxie « hégélienne ». En effet, l'influence de Croce sur ce système est clair. Bien entendu, ce n'est pas ici le moment d'entrer dans les détails, ni le lieu pour une discussion des nombreuses questions qu'ils soulèvent. Toutefois, certains détails valent la peine d'être soulignés. Je dois indiquer en premier lieu un point concernant le mouvement allant du flux des sensations à la conscience de premier niveau, par le biais de l'" attention". Cette idée est bien entendu issue de la psychologie $e^{43}$, mais on doit noter que Collingwood a, de manière comprimée, passé en revue l'ensemble de son système dans la partie I du New Leviathan, en faisant des modifications substantielles qui indiquent un changement d'orientation vers un point de vue de plus en plus linguistique:

[...] fixez votre esprit sur la question en débat, et vous verrez que l'acte pratique de nommer votre sentiment, c'est ce qui enclenche votre conscience que vous en avez (Collingwood, 1992, 6.27).

Il ne peut donc y avoir aucun niveau pré-linguistique autre que celui du pur «flux des sensations », et toute conscience est déjà une conscience de nature linguistique. Il n'y a donc aucune place pour des émotions qui seraient conscientes sans être exprimées linguistiquement.

40. Il est cependant clair, partant de ce qu'il dit ailleurs dans le livre ainsi que dans The New Leviathan, que Collingwood concevait vraiment cette "activité de conscience» comme étant de nature linguistique.

41. Ce fait est souligné par Davies, 2008, 173.

42. C’est aussi la cause des nombreuses difficultés soulignées dans Davies, 2008.

43. Cf. infra, note 36 . 
Deuxièmement, une des particularités de la notion de langage de Collingwood est qu'elle est plutôt large et qu'elle ne couvre pas seulement le discours mais aussi

[...] tout système de mouvements corporels, non nécessairement vocal, par lesquels les hommes qui les produisent veulent dire ou signifient quelque chose (Collingwood, 1992, 6.1) ${ }^{44}$.

C'est pourquoi il dit de la danse qu'elle est la «mère de tous les langages » (Collingwood, 1938, 244 et 246). Placée où il est, au stade le plus primitif des niveaux de conscience exposés plus haut, l'art devient omniprésent, et si l'" activité artistique» est une activité d' "expression des émotions" (dans le sens spécifique exposé plus haut), alors l'art est tout simplement co-extensif avec le langage comme nous venons de le définir (Collingwood, 1938, 273 et 275). Cela est également évident de par le fait que les conceptions de Collingwood ne laissent aucune place à une conception de l'art dans laquelle l'œuvre d'art est - en comprimant (a) et (c) un état interne de l'artiste qui pourrait ne pas être externalisé: dans le cas de la danse, c'est clairement impossible. Comme Collingwood l'a lui-même souligné, cela ne signifie pas, bien entendu, qu'il ne puisse y avoir aucun cas dans lequel l'œuvre d'art est d'abord un état purement interne, par exemple dans le cas d'un poème imaginé par le poète simplement en pensée. Mais cela nous montre seulement que Collingwood n'affirme pas une thèse essentialiste sur l'art. Il n'affirme pas, par exemple dans les passages cités plus haut (Collingwood, 1938, 151), que l'œuvre d'art est toujours d'abord un état interne de l'artiste, mais seulement qu'elle peut l'être.

Troisièmement, une autre raison fait que Wollheim ne peut avoir raison dans le portrait qu'il dresse de Collingwood et qui relève du fait que l' «art est langage " ${ }^{45}$. Dit brièvement, cela signifie que les conditions de la compréhension linguistique s'appliquent mutatis mutandis au cas de l'interprétation de l'œuvre d'art. C'est pourquoi on retrouve dans The Principles of Art un long chapitre sur le langage et la compréhension (Collingwood, 1938, 225-229) qui avance une explication de la possibilité de partager une émotion par le biais de l'art. Dans ce chapitre, Collingwood développe une conception hautement originale du langage et souligne que la conscience de soi émerge conjointement avec la conscience des autres et que cette découverte est la découverte du soi à la fois en tant que locuteur et en tant qu'auditeur:

44. Voir aussi Collingwood, 1938, 243.

45. Incidemment, c'est une thèse que Wollheim lui-même rejette (Wollheim, 1987, 44). Rappelons-nous que, selon lui, «on retrouve dans la peinture le type d'explication qui a été évacuée — et plutôt avec raison — du champ du langage sous l'influence de Wittgenstein » (Wollheim, 1987, 22). Une re-psychologisation de l'esprit est au menu d'ouvrages tels que Wollheim, 1993 et 1999. Ce serait un peu injuste de dépeindre Collingwood comme étant «dépassé » sur ce point. 
La conscience ne commence pas comme une simple conscience de soi, [...] pour ensuite procéder, par un processus quelconque [...] à la construction ou à l'inférence d'autres personnes. Chacun de nous est un être fini, entouré par d'autres du même genre; et la conscience de notre propre existence est également la conscience de l'existence de ces autres. [...] La découverte de soi comme personne par l'enfant est également la découverte de soi comme membre d'un monde de personnes [...]. La découverte de ma propre personne est la découverte que je peux parler, que je suis une personne ou un locuteur. En parlant, je suis à la fois le locuteur et l'auditeur, et puisque la découverte de ma propre personne est également la découverte d'autres personnes autour de moi, c'est la découverte de locuteurs et d'auditeurs autres que moi. Ainsi, l'expérience du discours contient en elle-même d'emblée, en principe, les expériences de parler aux autres et d'entendre les autres me parler (Collingwood, 1938, 248249).

La compréhension est donc le résultat d'un recentrement de l'auditeur comme locuteur, ce qui est possible sur la base du fait que le langage est dénué de toute asymétrie imposée par un quelconque privilège de la première personne:

L'expression [d'une émotion] est un discours, et le locuteur est son propre premier auditeur. En s'écoutant parler, il est conscient de lui-même comme possédant l'idée qu'il s'entend exprimer [...]. Nous décrivons [...] notre situation comme auditeurs de ce que nous disons nous-mêmes. La personne à laquelle est adressée le discours est déjà familiarisée avec cette double situation [...]. L'auditeur [...], conscient qu'une autre personne comme lui s'adresse à lui, considère ce qu'il entend comme si c'était son propre discours: il se parle à lui-même avec les mots qu'il entend et qui lui sont adressés, et construit ainsi en lui-même l'idée exprimée par ces mots. En même temps, conscient du locuteur comme personne différente de lui-même, il attribue l'idée à cette autre personne. Comprendre ce que quelqu'un vous dit, c'est donc lui attribuer l'idée que ses mots ont éveillé en vous; et cela implique de traiter ces mots comme étant les vôtres (Collingwood, 1938, 249-250).

L'auditeur comprend celui qui parle de la même manière qu'il se comprend lui-même et, en même temps, il attribue l'idée qu'il comprend à celui qui parle. Les conceptions exposées dans ces riches passages méritent certainement une étude critique. Mais, pour les fins de cet article, il suffit que le lecteur réalise que la position de Collingwood est appuyée par une conception hautement originale du langage. Cette conception a éte saluée par des philosophes analytiques contemporains aussi diversifiés que Simon Blackburn, qui a vu en lui, à mon avis à tort, un précurseur de la théorie moderne de la simulation en psychologie (Blackburn, 1992) et Donald Davidson, qui y a vu un précuseur de sa propre "triangulation » (Davidson, 2001, 219). Il n'est pas important de décider ici qui a raison, mais une chose est certaine: on ne peut pas dire que sa conception était rétrograde pour les années 1930. C'est en fait exactement le contraire, tant et si bien que je dois insister encore une fois sur le fait qu'il n'y a aucun équivalent de tout cela 
du côté des conceptions de Croce et de Bosanquet. Non seulement il est clair que (a) - (c) ne caractérisent pas sa position, mais il serait tout simplement faux d'affirmer (d) et de dire que, selon Collingwood, le lien est rompu entre l'artiste et son spectateur. C'est sur ce point que je vais insister dans mes remarques finales.

\section{Collingwood et le rôle social de l'art}

Pour conclure, j'aimerais revenir sur le rôle social de l'art. Comme je l'ai souligné, Collingwood cherchait à faire jouer à l'art un rôle social essentiel, celui d'une "médecine communautaire contre la pire maladie de l'esprit, la corruption de la conscience» (Collingwood, 1938, 336). Les historiens de la philosophie véhiculent souvent l'idée erronée que les philosophes, raisonnant comme ils le font dans des sphères éthérées et éternelles, ne sont jamais conditionnés par leur contexte. Collingwood, qui, avec raison, jugeait cette idée absurde, nous dit dans la préface de Principles of Art:

Tout ce qui a été écrit dans ce livre l'a été dans l'espoir que cela aura un effet pratique, direct ou indirect, sur la situation de l'art en Angleterre en 1937 (Collingwood, 1938, 279).

Le livre fut publié à la veille de l'accord de Munich de 1938, à une époque où Collingwood, qui avait vécu jusque-là la vie de réclusion typique d'un professeur d'Oxford, devint de plus en plus préoccupé par la situation politique (certains des partisans de l'accord de Munich et de "l'accommodement » étaient ses collègues à Oxford, ce qui le troubla énormément ${ }^{46}$. Le contexte de "la condition de l'art en Angleterre en 1937 » est également celui d'un pays dont les citoyens ne veulent pas reconnaître que leur tâche est de réagir à la montée du nazisme et du fascisme, de ne pas laisser tomber la république espagnole, etc. Il croyait qu'un des défauts les plus graves des démocraties était, en contraste avec les états totalitaires, leur inaptitude à susciter un appui émotionnel, et c'est cela qu'il exprimait en parlant d'une «corruption de la conscience» sur le plan communautaire. Les citoyens britanniques lui semblaient incapables de cette sorte d'émotion nécessaire à une vie démocratique saine, et il croyait que ce défaut était en partie dû à l'état désolant des arts dans son pays. L'art aurait dû amener le type d'émotions nécessaires au soutien de la démocratie dans l'espace public au moment où un conflit décisif se tramait, mais il n'a pas réussi à le faire. Il croyait donc que c'est la tâche de l'artiste que de prendre ici les rênes et de réussir à exprimer une émotion qui soit partagée par la communauté, un «sentiment commun » comme l'aurait dit Bosanquet. Au fond, donc, la philosophie de l'art de Collingwood est intimement liée à sa philosophie politique. Cette remarque l'illustre bien:

46. La philosophie politique de Collingwood est exposée dans The New Leviathan (Collingwood, 1992) et dans des essais réunis dans Collingwood, 1989. Pour une discussion de celle-ci, voir Boucher, 1989. 
Il me semble donc évident que, selon mes propres prémisses, un artiste avec de forts sentiments et convictions politiques sera dans cette mesure mieux qualifié pour produire des œuvres d'art qu'un artiste qui n'en a pas. (Collingwood, 1938, 279).

Comme je l'ai souligné, pour être en mesure de tenir ces propos, il avait besoin d'une explication crédible de la possibilité que les membres d'une communauté puissent partager une émotion par le biais d'une œuvre d'art. Il affirma d'abord, de manière plutôt réaliste, que l'émotion qui est "mise dans l'œuvre d'art» par l'artiste et l'émotion qui est ressentie par le spectateur sont identiques:

Si l'artiste peint son tableau de telle manière que, lorsque nous le regardons en utilisant notre imagination, nous fassions une expérience comme celle qu'il a faite en le peignant, il n'y aurait pas grand sens à dire que nous apportons cette expérience avec nous et que nous ne la trouvons pas dans le tableau. Si nous disions cela à l'artiste, il rirait de nous et nous assurerait que ce que nous avons cru lire dans le tableau était tout simplement ce qu'il y avait mis (Collingwood, 1938, 150) ${ }^{47}$.

C'est certainement là une thèse controversée tellement elle va à l'encontre des sensibilités post-modernes de notre époque. Elle était en outre appuyée par des arguments sur la possibilité pour l'historien de repenser (re-enact) la pensée de l'agent historique qu'il étudie par une pensée identique à celle-ci, arguments que Collingwood a développés dans sa philosophie de l'histoire ${ }^{48}$. (Peut-être qu'une théorie de la vérité comprise comme identité est présupposée $\mathrm{ici}^{49}$.) Ce qui est peut-être plus intéressant, c'est qu'il y a un argument dérivé de sa philosophie du langage, que nous avons brièvement esquissée plus haut à l'aide de quelques citations. Comme nous l'avons vu, Collingwood considérait le langage comme dénué de toute forme d'asymétrie entre l'auditeur et le locuteur, de telle sorte qu'ils peuvent changer de place. Cette conception est simplement étendue à l'art, où l'interchangeabilité des perspectives entre l'artiste et le spectateur est une condition préalable à la compréhension. La position de Collinwood sur les émotions devient donc réellement «linguistique»:

La relation entre le locuteur et l'auditeur, en tant que deux personnes distinctes, en est une qui est facilement mal comprise en raison de sa grande familiarité. Nous sommes capables de la penser comme une relation dans laquelle le locuteur "communique» son émotion à l'auditeur. Mais les émotions ne peuvent être partagées comme on partage un repas ou un breuvage, ou distribuées comme des vêtements usés [...]. Indépendamment du langage, ni lui, ni moi, ni aucune tierce personne peut comparer ses émotions avec les miennes, comme pour voir si elles sont semblables ou non. Lorsqu'on parle

47. Voir également Collingwood, 1938, 308.

48. Voir Saari, 1989, et D’Oro, 2000, sur cette question.

49. Pour de plus amples informations, voir Baldwin, 1991. 
d'une telle comparaison, on parle de quelque chose qui est fait en utilisant le langage; ainsi, la comparaison doit être définie en termes d'actes de parler et d'entendre, et non l'inverse (Collingwood, 1938, 249).

La même chose est valable pour l'art:

Si ce [que l'artiste] souhaite faire est d'exprimer ses émotions de manière intelligible, il doit les exprimer de manière telle qu'elles lui soient intelligibles. Son auditoire est alors dans la position de personnes qui l'auraient entendu par hasard (Collingwood, 1938, 111).

Si quelqu'un dit "deux et deux font quatre » en présence de quelqu'un qui est incapable de réaliser même les opérations arithmétiques les plus simples, il se comprendra lui-même, mais il ne sera pas compris par son auditeur. L'auditeur ne peut comprendre que s'il peut additionner deux et deux dans son esprit. Cela ne fait aucune différence qu'il puisse ou non le faire avant d'avoir entendu le locuteur prononcer ces mots. Ce qu'on dit ici à propos de l'expression des pensées est aussi vrai à propos de l'expression des émotions. Si par exemple un poète exprime une certaine peur, les seuls auditeurs qui pourront le comprendre sont ceux qui sont eux-mêmes capables de faire l'expérience de ce genre de peur. Ainsi, lorsque quelqu'un lit et comprend un poème, il ne fait pas que simplement comprendre l'expression de [ses propres] émotions par le poète, il exprime ses propres émotions dans les mots du poète, qui sont ainsi devenus ses propres mots. Nous savons qu'il exprime ses émotions par le fait qu'il nous permet d'exprimer les nôtres (Collingwood, 1938, 118).

Cette conception a de profondes conséquences. Principalement, c'est l'idée que l'artiste et le spectateur collaborent, que l'œuvre d'art est donc le produit d'une collaboration, c'est-à-dire que, tout comme pour la signification linguistique, l'expérience de partager une émotion n'appartient jamais uniquement à l'un ou l'autre. Cette conception, qui relève encore aujourd'hui de l'avant-garde, est clairement une conséquence de sa philosophie du langage:

L'activité esthétique est l'activité de parler. Le discours est discours seulement dans la mesure où il est parlé et entendu. Il ne fait aucun doute qu'un homme peut se parler à lui-même et être son propre auditeur; mais ce qu'il se dit peut en principe être dit à quiconque partage son langage. En tant qu'être fini, un homme devient conscient de lui-même en tant que personne seulement dans la mesure où il se trouve en relation à d'autres qu'il perçoit simultanément comme personnes. Et il n'y a aucun moment dans la vie d'un homme où il perdra conscience de lui-même comme personne. [...] S'il a une nouvelle émotion, il doit l'exprimer aux autres de telle manière qu'il peut, en les reconnaissant aptes à la partager, s'assurer que sa conscience n'est pas corrompue.

Cela ne contredit pas la doctrine affirmée ailleurs dans ce livre selon laquelle l'expérience ou activité esthétique est quelque chose qui a lieu dans l'esprit de l'artiste. L'expérience d'être entendu est une expérience qui a lieu dans l'esprit du locuteur, bien qu'un auditeur soit nécessaire à son existence et qu'ainsi l'activité soit une collaboration. L'amour mutuel est une activité de collaboration: mais l'expérience de cette activité dans l'esprit de chaque amant pris 
singulièrement est une expérience différente de celle d'aimer et d'être rejeté (Collingwood, 1938, 317-318).

Enfin, on doit souligner que Collingwood croyait réellement que l'art est une activité qui appartient à la communauté, et non à un individu isolé:

[L'activité artistique] est une activité collective qui n'appartient pas à un être humain mais à une communauté. Elle est réalisée non seulement par l'homme que nous appelons, de manière individualiste, l'artiste, mais en partie par tous les autres artistes dont nous disons qu'ils l'ont influencé, là où nous voulons dire en fait qu'ils ont vraiment collaboré avec lui. L'activité artistique est réalisée non pas uniquement par ce collectif d'artistes, mais (dans le cas des arts de performance) par les exécutants, qui n'agissent pas simplement sous les ordres de l'artiste, mais qui collaborent avec lui afin de produire l'œuvre achevée. Et même là, l'activité de la création artistique n'est pas complète; car il doit y avoir une audience dont la fonction n'est pas simplement réceptive mais également collaborative. L'artiste (bien qu'il puisse tenter de le nier sous l'influence de préjugés individualistes) est ainsi en relation de collaboration avec une communauté entière; non pas une communauté idéale de tous les êtres humains, mais la communauté actuelle des artistes auxquels il a emprunté, des exécutants qu'il emploie, et de l'audience à laquelle il s'adresse (Collingwood, 1938, 324).

Cette conception a plusieurs implications controversées, et certaines d'entre elles ont été exposées par Collingwood, par exemple la nécessité d'abolir la loi sur les droits d'auteur (Collingwood, 1938, 325-326), son apologie de l'avant-garde et du Group Theatre de tendance plutôt gauchiste à Londres (Collingwood, 1938, 329), son hommage aux poètes et dramaturges Louis MacNiece et T. S. Eliot (Collingwood, 1938, 332-335) ${ }^{50}$, ou ce simple commentaire, qu'on croirait avoir été écrit il y a quelques mois :

Nous devons faire face au fait que chaque performeur est nécessairement un coauteur, et nous devons développer ces implications (Collingwood, 1938, 328).

Encore une fois, ce n'est pas ici le lieu de discuter de ces questions, mais ces remarques pourront au moins servir d'illustration de l'originalité, de la radicalité et de la modernité des conceptions de Collingwood. Et, à tout le moins, le lecteur aura noté à quel point nous nous sommes ainsi éloigné de la prétendue "théorie de Croce et de Collingwood », puisque rien de cela ne fait sens à la lumière de ma reconstruction de la philosophie de Collingwood. Il n'est pas seulement faux de dire que le lien entre l'artiste et le spectateur est rompu chez Collingwood; cela est à l'inverse de la vérité, car il a plaidé en faveur de ce lien de la manière la plus originale qui soit pour son époque! Pendant plusieurs générations, l'idée même d'une "théorie

50. Le fait que la théorie de Collingwood était censée apporter un soutien à l'avantgarde artistique de son temps a même été utilisé contre lui dans A Philosophy of Mass Art de Noël Carroll (Carroll, 1998, 102)! 
de Croce et de Collingwood» fut un prétexte pour ne pas lire ce philosophe qui aurait dû plutôt piquer notre curiosité; celui-ci mérite certainement d'être entendu, à condition de ne pas se laisser guider par toutes sortes de préjugés ${ }^{51}$.

Traduit de l'anglais par Guillaume Fréchette

\section{Bibliographie}

Alexander, S. Beauty and other Forms of Value, Londres, MacMillan, 1933.

-. Philosophical and Literary Pieces, Londres, MacMillan, 1939.

Anderson, D. R. «Collingwood, Robin George », in M. Kelly (dir.) Encyclopedia of Aesthetics, Oxford, Oxford University Press, vol. 1, 1998, 393-395.

Baldwin, T. «The Identity Theory of Truth ", Mind, vol. 100, 1991, 35-52.

Baxandall, M. Formes de l'intention. Sur l'explication historique des tableaux, Nîmes, Jacqueline Chambon, 1991.

Bell, C. Art, Londres, Chatto \& Windus, 1914.

Blackburn, S. «Theory, Observation and Drama », Mind and Language, vol. 7, 1992, 187-203.

Bosanquet, B. A History of Aesthetics, Londres, Allen \& Unwin, 1904.

-. Three Lectures on Aesthetic, Londres, Macmillan, 1915.

—. "Croce's Aesthetic», Proceedings of the British Academy, vol. 9, 1920a, 1-28.

- _. "Croce's Aesthetic ", Mind, vol. 29, 1920b, 212-215.

Boucher, D. The Social and Political Thought of R. G. Collingwood, Cambridge, Cambridge University Press, 1989.

Carr, H. W. «Mr. Bosanquet on Croce's Aesthetic », Mind, vol. 29, 1920, 208-211.

Carritt, E. F. (dir.) Philosophies of Beauty from Socrates to Robert Bridges, being the Sources of Aesthetic Theory, Oxford, Clarendon Press, 1931.

- What is Beauty? A First Introduction to the Subject and to Modern Theories. Oxford, Clarendon Press, 1932.

—. An Introduction to Aesthetics, Londres, Hutchinson, 1949.

- «Croce and his Aesthetic», Mind, vol. 62, 452-464, 1953.

Carroll, N. A Philosophy of Mass Art, Oxford, Clarendon Press, 1998.

Collingwood, R. G. Speculum Mentis or The Map of Knowledge, Oxford, Clarendon Press, 1924.

- Outlines of a Philosophy of Art, Oxford, Clarendon Press, 1925.

- The Principles of Art, Oxford, Oxford University Press, 1938.

- Essays in the Philosophy of Art, A. Donagan (dir.), Bloomington, Indiana University Press, 1964.

51. Une version antérieure de cet article a été lue au colloque "Comment être idéaliste? Bradley et Collingwood " qui a eu lieu à l'Université Blaise Pascal de Clermont-Ferrand en juin 2006 ainsi qu'à une réunion du British Idealism Specialist Group de la Political Science Association de Grande-Bretagne à Gregynog, au Pays de Galles, en décembre 2007. J'aimerais remercier les participants à ces deux activités pour leurs commentaires, tout comme David Boucher, James Connelly, David Davies, Giuseppina D’Oro, Sébastien Gandon, Laurent Jaffro et Bill Manders pour leur aide et leurs commentaires qui ont fait leur chemin dans cet article, depuis ces dernières années. J'aimerais aussi remercier Mathieu Marion pour son aide dans la version anglaise de cet article et pour plusieurs commentaires détaillés qui l'ont grandement amélioré. J'ai pu largement bénéficier de sa connaissance approfondie de la philosophie britannique. Il a en outre vérifié la traduction de Croce, de l'italien au français. 
- Essays in the Philosophy of History, Austin TX, University of Texas Press, 1965.

—. Essays in Political Philosophy, Oxford, Clarendon Press, 1989.

- The New Leviathan, $2^{\mathrm{e}}$ édition, Oxford, Clarendon Press, 1992; traduction française: Le Nouveau Léviathan, Paris, Kimé, 2001.

- The Idea of history, $2^{\mathrm{e}}$ édition, Oxford, Clarendon Press, 1994.

—. An Essay on Philosophical Method, Oxford, Clarendon Press, 2005.

- "A Selection of Collingwood's Writings on Aesthetics, compiled and introduced by James Connelly ", Collingwood Studies, vol. 2, 1995, 182-212.

—. "Aesthetic and the Mind, introduced by James Connelly", Collingwood Studies, vol. 3, 1996, 188-193.

Cometti, J.-P., J. Morizot et R. Pouivet. Questions d'esthétique, Paris, P. U. F., 2000. Croce, B. The Philosophy of Giambattista Vico, Londres, Howard Latimer, 1913.

-. Aesthetics as Science of Expression and General Linguistics, $2^{\mathrm{e}}$ édition, Londres, MacMillan, 1922.

—. Estetica come scienza dell'espressione e linguistica generale, Bari, Laterza \& Figli, 1958.

- The Aesthetics as the Science of Expression and Linguistic in General, Cambridge, Cambridge University Press, 1992.

- Thèses fondamentales pour une esthétique comme science de l'expression et linguistique générale, Nîmes, Champ social éditions, 2006.

Davidson, D. Subjective, Intersubjective, Objective, Oxford, Clarendon Press, 2001.

Davies, D. Art as Performance, Oxford, Blackwell, 2004.

Davies, D. "Collingwood's 'Performance' Theory of Art », British Journal of Aesthetics, vol. 48, 2008, 162-174.

Dewey, J. 1893. "Review of A History of Aesthetics by Bernard Bosanquet ", Philosophical Review, vol. 2, 63-69.

Dewey, J. Art as Experience, New York NY, Minton, Balch \& Co, 1934.

Donagan, A. "The Croce-Collingwood Thoery of Art ", Philosophy, vol. 33, 1958, 162-167.

—. The Later Philosophy of R. G. Collingwood, Oxford, Clarendon Press, 1962.

—. "Collingwood's Debt to Croce ", Mind, vol. 81, 1972, 265-266.

D'Oro, G. "Collingwood on Re-Enactment and the Identity of Thought ", Journal of the History of Philosophy, vol. 38, 2000, 87-101.

Elton, W. (ed.) Aesthetics and Language, Oxford, Blackwell, 1959.

Gallie, W. B. "The Function of Philosophical Aesthetics», in W. Elton (dir.), Aesthetics and Language, Oxford, Blackwell, 1959, 13-35.

Goodman, N. (168), Languages of Art. An Approach to the Theory of Symbols, Indianapolis IN, Bobbs-Merrill; traduction française: Langages de l'art, Paris, Hachette, 2005.

Gombrich, E. H. Art and Illusion. A Study in the Psychology of Pictorial Representation, $2^{\mathrm{e}}$ édition, Princeton, Princeton University Press, 1961; traduction française: L'art et l'illusion. Psychologie de la représentation picturale, Paris, Gallimard, 1971.

Hospers, J. «The Croce-Collingwood Theory of Art », Philosophy, vol. 31, 1956, 291-308.

Inglis, F. History Man. The Life of R. G. Collingwood, Princeton NJ, Princeton University Press, 2009. 
Kemp, G. "The Croce-Collingwood Theory as Theory », Journal of Aesthetics and Art Criticism, vol. 61, 2003, 171-193.

Knox, T. M. "Review of The Formative Years of R. G. Collingwood by William Johnston ", Philosophical Quarterly, vol. 19, 1969, 165-166.

Lang, B. "Bosanquet's Aesthetic: A History and Philosophy of the Symbol», The Journal of Aesthetics and Art Criticism, 26, 1968, 377-387.

Levinson, J. The Pleasures of Aesthetics: Philosophical Essays, Ithaca NY, Cornell University Press, 1996.

- Contemplating Art, Oxford, Clarendon Press, 2006.

Mabbott, J. Oxford Memories, Oxford, Thornton's, 1986.

Mauro, T. de. Une introduction à la sémantique, Paris. Payot, 1969.

Michaud, Y. Critères esthétiques et jugement de goût, Nîmes, Jacqueline Chambon, 1999.

Mink L. O. Mind, History and Dialectic. The Philosophy of R. G. Collingwood, Bloomington IN, Indiana University Press, 1969.

Mulhall, S. «Expression », in D. Cooper (dir.), A Companion to Aesthetics, Oxford, Blackwell, 1992, 144-149.

Murdoch, I. The Sovereignty of Good over other Concepts, Londres, Cambridge University Press, 1967.

Passmore, J. «G. F. Stout's Editorship of Mind (1892-1920) », Mind, vol. 85, 1976, 17-36.

Read, H. Art and Society, New York, Schocken Books, 1966.

Reid, L. A. A Study of Aesthetics, Londres, Allen \& Unwin, 1931.

Ridley, A. "Not Ideal: Collingwood's Expression Theory ", Journal of Aesthetics and Art Criticism, vol. 55, 1997, 263-72.

- R. G. Collingwood: A Philosophy of Art, Londres, Phoenix, 1998.

Saari, H. "R. G. Collingwood on the Identity of Thoughts", Dialogue, vol. 28, 1989, 77-89.

Saw, R. L. "E. F. Carritt ", British Journal of Aesthetics, vol. 4, 1963, 3-6.

Sclafani, R. "Wollheim on Collingwood», Philosophy, vol. 51, 1976, 353-359.

Spadoni, C. "Bertrand Russell on Aesthetics », Russell, n.s., vol. 4, 1984, 49-82.

Sully, J. "Review of A History of Aesthetics by Bernard Bosanquet ", Mind, n.s., vol. 2, 1893, 110-117

Sweet, W. «British Idealist Æsthetics: Origins and Themes », Bradley Studies, vol. 7, 2001, 131-161.

Vico, G. La science nouvelle (1972), Paris, Gallimard, 1993.

Ward, J. «Psychology", Encyclopaedia Britannica, $9^{e}$ édition, 1886, vol. XX, 37-85.

—. Psychological Principles, Cambridge, Cambridge University Press, 1918.

Weitz, M. "The Role of Theory in Æsthetics", Journal of Æsthetics and Art Criticism, vol. 15, 1956, 27-35.

Wollheim, R. F. H. Bradley, Londres, Penguin Books, 1959.

- . "On an Alleged Inconsistency in Collingwood's Aesthetic », in M. Krausz (dir.), Critical Essays in the Philosophy of R. G. Collingwood, Oxford, Clarendon Press, 1972, 68-78.

- On Art and the Mind, Cambridge MA, Harvard University Press, 1974.

- Art and its Objects, $2^{\mathrm{e}}$ édition, Cambridge, Cambridge University Press, 1980.

—. Painting as an Art, Princeton, NJ, Princeton University Press, 1987.

- The Mind and its Depths, Cambridge MA, Harvard University Press, 1993.

—. On the Emotions, New Haven CT, Yale University Press, 1999. 